\title{
The Magnetic Field of Active Region 11158 During the 2011 February 12-17 Flares : Differences between Photospheric Extrapolation and Coronal Forward-Fitting Methods
}

\author{
Markus J. Aschwanden ${ }^{1}$ \\ $\left.{ }^{1}\right)$ Lockheed Martin Advanced Technology Center, Org. A021S, Bldg.252, 3251 Hanover St., Palo Alto, CA \\ 94304, USA; e-mail: aschwanden@lmsal.com \\ and \\ Xudong Sun ${ }^{2}$ and Yang Liu ${ }^{2}$ \\ $\left.{ }^{2}\right)$ W. W. Hansen Experimental Physics Laboratory, Stanford University, Stanford, CA 94305, USA; \\ e-mail: xudongs@stanford.edu,yliu@sun.stanford.edu
}

\begin{abstract}
We developed a coronal non-linear force-free field (COR-NLFFF) forward-fitting code that fits an approximate non-linear force-free field (NLFFF) solution to the observed geometry of automatically traced coronal loops. In contrast to photospheric NLFFF codes, which calculate a magnetic field solution from the constraints of the transverse photospheric field, this new code uses coronal constraints instead, and this way provides important information on systematic errors of each magnetic field calculation method, as well as on the non-forcefreeness in the lower chromosphere. In this study we applied the COR-NLFFF code to active region NOAA 11158, during the time interval of 2011 Feb 12 to 17, which includes an X2.2 GOES-class flare plus 35 $\mathrm{M}$ and C-class flares. We calcuated the free magnetic energy with a 6 -minute cadence over 5 days. We find good agreement between the two types of codes for the total nonpotential $E_{N}$ and potential energy $E_{P}$, but find up to a factor of 4 discrepancy in the free energy $E_{\text {free }}=E_{N}-E_{P}$, and up to a factor of 10 discrepancy in the decrease of the free energy $\Delta E_{\text {free }}$ during flares. The coronal NLFFF code exhibits a larger time variability, and yields a decrease of free energy during the flare that is sufficient to satisfy the flare energy budget, while the photospheric NLFFF code shows much less time variability and an order of magnitude less free energy decrease during flares. The discrepancy may partly be due to the pre-processing of photospheric vector data, but more likely due to the non-forcefreeness in the lower chromosphere. We conclude that the coronal field cannot be correctly calculated based on photospheric data alone, but requires additional information on coronal loop geometries.
\end{abstract}

Subject headings: Sun: Corona — Magnetic fields — Sun: UV radiation

\section{INTRODUCTION}

The art of computing solar magnetic fields has evolved from routine potential field (PF) extrapolations to nonlinear force-free field (NLFFF) computations. The most important aspect concerning these two types of magnetic field models is the difference of magnetic energy content between them, the so-called free energy, which represents the maximum energy that can be dissipated during a magnetic instability that is driving 
solar flares, coronal mass ejections, or other eruptive events. Monitoring the magnetic evolution in active regions became an efficient diagnostic to measure the emergence of new magnetic flux on the solar surface, the magnetic storage, the injection of electric currents and helicity, the step-wise dissipation of nonpotential energy during flares, the energy conversion of magnetic energy during a magnetic reconnection process into particle acceleration, etc. The quantification of the free magnetic energy probably provides the strongest constraint to discriminate cause and consequence in the various energy conversion processes. It is therefore imperative to develop accurate and efficient tools to measure and monitor the evolution of magnetic energy in solar active regions. In this study we explore a novel method to calculate an approximate NLFFF solution using an automated loop tracing code, which aims to find force-free magnetic field solutions that are most consistent with the observed geometry of coronal loops, such as observed in high-resolution extremeultraviolet (EUV) images from the Atmospheric Imaging Assembly (AIA) (Lemen et al. 2012) onboard the Solar Dynamics Observatory (SDO) spacecraft (Pesnell et al. 2012).

NLFFF Modeling has been applied to active regions (Bobra et al. 2008; Su et al. 2009a; Inoue et al. 2011, 2013; Savcheva et al. 2012a), to coronal sigmoids (Savcheva et al. 2012b, 2012c; Inoue et al. 2012), to photospheric magnetic field changes (Liu et al. 2012), to magnetic flux emergence and energy build-up before flares (Su et al. 2009b; Li et al. 2007), to flares (Guo et al. 2008; Schrijver et al. 2008), to confined eruptions (Guo et al. 2010), to Coronal Mass Ejections (CMEs) (Su et al. 2011; Feng et al. 2013), as well as to MHD simulations (Savcheva et al. 2012a). Earlier studies compared theoretical models with observed images in a rather qualitative way, but recent studies go into quantitative comparisons of free energies and statistics of misalignment angles between modeled field lines and observed coronal loop geometries. Comparisons of up to 14 different NLFFF codes revealed substantial differences in the amount of calculated free magnetic energies, ranging from an (unphysical) minumun value $E_{N} / E_{P}=0.88$ below unity, to a maximum of $E_{N} / E_{P}=1.30$ for an X3.4-class flare (Schrijver et al. 2008). An evaluation of current NLFFF modeling was conducted in a study of the solar active region NOAA 10953 (2007 April 30) with a dozen of different NLFFF codes, which led to three major requirements for successful NLFFF modeling: (1) a sufficiently large field-of-view of the vector magnetic field data and the computation box, (2) accomodation of uncertainties in the boundary data, and (3) a realistic model of the transition from the non-forcefree photosphere to the force-free base of the corona (DeRosa et al. 2009). A pre-processing step to minimize the net force and torque in the photospheric boundary data was suggested (Wiegelmann et al. 2006, 2008; Wheatland and Regnier 2009), which improved the extrapolation above the chromosphere, but the field connectivity and free magnetic energy were not well recovered (Metcalf et al. 2008; Yamamoto et al. 2012; Jiang and Feng 2013). The latter issue is manifested in a major discrepancy between the computed magnetic field lines and stereoscopically observed geometries of coronal loops (Fig. 1), amounting to 3D misalignment angles of $\approx 24^{\circ}-44^{\circ}$ (DeRosa et al. 2009). This mismatch angle can be reduced by forward-fitting of parameterized potential field models (Aschwanden and Sandman 2010; Sandman and Aschwanden 2011), by forward-fitting using a quasi-Grad-Rubin NLFFF method (Malanushenko et al. 2011, 2012), and by forward-fitting of analytical NLFFF approximations (Aschwanden et al. 2012, Aschwanden 2013a, 2013b, 2013c; Aschwanden and Malanushenko 2013). The latter method has been demonstrated to work equally well for both 3-dimensional (3D) loop coordinates, obtained with stereoscopy, and 2-dimensional (2D) loop projections (Aschwanden 2013c).

In this study we developed our forward-fitting code with analytical NLFFF approximations further by (1) implementing an automated loop tracing code for detection of coronal loops in multi-wavelength EUV images, which makes the manual or visual loop tracing unnecessary (Aschwanden 2010), (2) by optimization of the forward-fitting technique to 2D loop coordinates, which relinquishes 3D reconstruction with stereoscopy (Aschwanden 2013c), and (3) by improved detection and rejection of false loop structures, caused by CCD 
pixel bleeding, CCD saturation, and diffraction patterns from by the EUV telescope entrance filter. The content of this paper is a brief analytical description of the method (Section 2), a brief description of the numerical code (Section 3), and data analysis of NOAA active region 11158 observed with SDO/AIA and HMI during the 2011 February 12-17 flares (Section 4), discussion and comparison with previous studies on the same active region and X-class flare (Section 5), and conclusions (Section 6).

\section{ANALYTICAL DESCRIPTION OF MAGNETIC MODELING}

Our Coronal Nonlinear Force-Free Field (COR-NLFFF) forward-fitting code consists of the following three major tasks (Fig. 2): (1) Automated loop tracing in coronal 2D images; (2) decomposition of a line-of-sight magnetogram into (sub-photospheric) magnetic charges that provide a parameterization of the potential and non-potential field; and (3) forward-fitting of the nonlinear force-free field approximation to the observed, automatically traced 2D loop coordinates. A brief theoretical description of these three modeling steps is provided in the following.

\subsection{Automated Loop Tracing}

An early version of the automated loop tracing code, called Oriented Coronal CUrved Loop Tracing (OCCULT), has been quantitatively compared with four other codes (Aschwanden et al. 2008). The method is based on oriented-directivity tracing of curvi-linear features, but in contrast to other general featureextraction algorithms, it is customized to solar EUV and SXR images by taking advantage of the specific property that coronal loops have large curvature radii, compared with their widths. Essentially, an image is highpass-filtered to enhance curvi-linear features with a small width, which are then traced with a guiding criterion that is defined in terms of the local curvature radius within small directional changes along a traced loop segment. The performance of this code was systematically improved by optimizing the guiding criterion, using the first-order term of the loop direction in the first version (Aschwanden 2010), and using the secondorder term of the local curvature radius in the latest version OCCULT-2 (Aschwanden, De Pontieu, and Katrukha 2013b).

\subsection{Potential Field Computation}

For the computation of a potential field, a line-of-sight (LOS) magnetogram that samples one magnetic field vector component $B_{z}(x, y)$ is sufficient to calculate a $3 \mathrm{D}$ potential field in a given computation box. While standard potential-field codes, such as the widely used Potential Field Source Surface (PFSS) code, are based on the eigen function (spherical harmonic) expansion method originally developed by Altschuler

and Newkirk (1969), we need a parameterization that can also be used for forward-fitting of non-potential 3D magnetic field models. The simplest method that is suitable for this purpose is the decomposition of a potential field into uni-polar magnetic charges $j=1, \ldots, N_{m}$ that are buried in sub-photospheric locations $\left(x_{j}, y_{j}, z_{j}\right)$, with the field strength $B(r)$ decreasing with the square of the radial distance $r$, which can be superimposed by an arbitrary large number $N_{m}$ of magnetic charges,

$$
\mathbf{B}(\mathbf{x})=\sum_{j=1}^{N_{\mathrm{m}}} \mathbf{B}_{j}(\mathbf{x})=\sum_{j=1}^{N_{\mathrm{m}}} B_{j}\left(\frac{d_{j}}{r_{j}}\right)^{2} \frac{\mathbf{r}_{\mathbf{j}}}{r_{j}}
$$


where $r_{j}=\left[\left(x-x_{j}\right)^{2}+\left(y-y_{j}\right)^{2}+\left(z-z_{j}\right)^{2}\right]^{1 / 2}$ is the distance of an arbitrary coronal location $\mathbf{x}=(x, y, z)$ to the subphotospheric charge location $\left(x_{j}, y_{j}, z_{j}\right)$, while $d_{j}=1-\left[x_{j}^{2}+y_{j}^{2}+z_{j}^{2}\right]^{1 / 2}$ is the depth of the buried charge, and $B_{j}$ is the magnetic field strength at the solar surface in vertical direction above the buried charge. The square-dependence of the radial field component $B(r) \propto r^{-2}$ warrants that each magnetic charge fulfills Maxwell's divergence-free condition,

$$
\nabla \cdot \mathbf{B}=0
$$

which it is also true for the summed magnetic field according to Equation (1), because the linear superposition of divergence-free fields is divergence-free too, i.e., $\nabla \cdot \mathbf{B}=\nabla \cdot\left(\sum_{j} \mathbf{B}_{\mathbf{j}}\right)=\sum_{j}\left(\nabla \cdot \mathbf{B}_{\mathbf{j}}\right)=0$.

The decomposition of a LOS magnetogram $B_{z}(x, y)$ into a finite number $N_{m}$ of magnetic charges can simply be accomplished by iterative decomposition of local maxima of the field into individual magnetic charges, each one characterized by the four parameters $\left(B_{j}, x_{j}, y_{j}, z_{j}\right), j=1, \ldots, N_{m}$, by iterating subsequent subtractions of decomposed Gaussian-like magnetic charges until the residual map reaches some noise threshold. The procedure is demonstrated in detail in Aschwanden and Sandman (2010). Typically, a number of $N_{m} \approx 100$ magnetic sources is sufficient to obtain a realistic potential field model of a solar active region.

\subsection{Nonlinear Force-Free Field Computation}

A nonlinear force-free field (NLFFF) model needs to fulfill both the Maxwell's divergence-free (Equation 2 ), and the force-free condition,

$$
\frac{\mathbf{j}}{c}=\frac{1}{4 \pi}(\nabla \times \mathbf{B})=\alpha(\mathbf{x}) \mathbf{B} .
$$

where $\alpha(\mathbf{x})$ is a scalar function that varies in space, but is constant along a given field line, and the current density $\mathbf{j}$ is co-aligned and proportional to the magnetic field $\mathbf{B}$. An approximate analytical solution of Equations (2)-(3) was recently calculated for vertically twisted fields (Aschwanden 2013a) that can be expressed by a superposition of an arbitrary number of $N_{m}$ magnetic field components $\mathbf{B}_{j}, j=1, \ldots, N_{m}$,

$$
\mathbf{B}(\mathbf{x})=\sum_{m=1}^{N_{m}} \mathbf{B}_{j}(\mathbf{x}),
$$

where each magnetic field component $\mathbf{B}_{j}$ can be decomposed into a radial $B_{r}$ and an azimuthal field component $B_{\varphi}$,

$$
\begin{gathered}
B_{r}(r, \theta)=B_{m}\left(\frac{d^{2}}{r^{2}}\right) \frac{1}{\left(1+b^{2} r^{2} \sin ^{2} \theta\right)}, \\
B_{\varphi}(r, \theta)=B_{m}\left(\frac{d^{2}}{r^{2}}\right) \frac{b r \sin \theta}{\left(1+b^{2} r^{2} \sin ^{2} \theta\right)}, \\
B_{\theta}(r, \theta) \approx 0 \\
\alpha(r, \theta) \approx \frac{2 b \cos \theta}{\left(1+b^{2} r^{2} \sin ^{2} \theta\right)},
\end{gathered}
$$

where $(r, \varphi, \theta)$ are the spherical coordinates of a magnetic field component system $\left(B_{j}, x_{j}, y_{j}, z_{j}, \alpha_{j}\right)$ with a unipolar magnetic charge $B_{j}$ that is buried at position $\left(x_{j}, y_{j}, z_{j}\right)$, has a depth $d=1-\left(x_{j}^{2}+y_{j}^{2}+z_{j}^{2}\right)^{1 / 2}$, a vertical twist $\alpha_{j}$, and $r=\left[\left(x-x_{j}\right)^{2}+\left(y-y_{j}\right)^{2}+\left(z-z_{j}\right)^{2}\right]^{1 / 2}$ is the distance of an arbitrary coronal position $(x, y, z)$ to the subphotospheric location $\left(x_{j}, y_{j}, z_{j}\right)$ of the buried magnetic charge. The force-free $\alpha$ 
parameter can be expressed in terms of the parameter $b$ (Equation 8), which quantifies the number $N_{t w i s t}$ of full twist turns over a (loop) length $L$,

$$
b=\frac{2 \pi N_{\text {twist }}}{L} .
$$

This analytical approximation is divergence-free and force-free to second-order accuracy in the parameter $(b r \sin \theta)$ (Aschwanden 2013a), which is proportional to the force-free parameter $\alpha$ as defined by Equation (8). In the limit of vanishing vertical twist ( $\alpha \mapsto 0$ or $b \mapsto 0$ ), the azimuthal component vanishes, $B_{\varphi} \mapsto 0$, and the radial component degenerates to the potential-field solution of a unipolar magnetic charge, $B_{r} \mapsto B_{j}(d / r)^{2}$, which is simply a radial field that points away from the buried charge and decreases with the square of the distance. As a caveat, we should be aware that the analytical approximation expressed in Equations (5)-(6) implies a vertical twist axis that produces a horizontal azimuthal non-potential field component $B_{\varphi}$, while horizontal twist axes with the corresponding non-potential components $B_{\varphi}$ cannot be represented with this parameterization, such as a horizontally oriented filament (see also discussion on the Gold-Hoyle flux rope in Appendix A of Aschwanden 2013a). We will label the nonpotential magnetic field of our model with a vertical twist axis as $B_{N, \perp}$, in order to remind about this restriction.

\subsection{Computation of Free Magnetic Energy}

The free energy is generally defined as the difference between the potential and non-potential magnetic energy, integrated over the volume of the computation box. In our case, since we include only vertical twist

axes in our nonpotential model, the difference energy $E_{\perp}^{\text {free }}$ will be a lower limit to the total free energy $E^{\text {free }}$,

$$
E_{\perp}^{\text {free }}=E_{N, \perp}-E_{P}=\frac{1}{8 \pi}\left(\int B_{N, \perp}^{2}(\mathbf{x}) d V-\int B_{P}^{2}(\mathbf{x}) d V\right) .
$$

In order to obtain a first-order correction, we can consider a current in a semi-circular loop or filament. This current flows in vertical direction at the footpoint of a loop or filament, while it flows in horizontal direction near the apex. This twist axis of the field-aligned current $j_{\|}$has thus a relative direction $\theta$ with a cosine-dependence along the loop, which will also enter the resulting nonpotential azimuthal field component $B_{\varphi}$ and leads to an average underestimate (as a function of the inclination angle $\theta$ to the vertical) of

$$
\left\langle B_{\varphi, \perp}\right\rangle=\left\langle B_{\varphi} \cos (\theta)\right\rangle=\left\langle B_{\varphi}\right\rangle\left(\frac{2}{\pi}\right) .
$$

The free energy $E_{\text {free }}$ scales with the square of the azimuthal (nonpotential) field component $B_{\varphi}$ by definition (Equation 10 in Aschwanden 2013a), and thus the average free energy $E_{\perp}^{\text {free }}$ obtained in a model that only includes electric currents associated with a vertical axis is

$$
E_{\perp}^{\text {free }}=E^{\text {free }}\left\langle\cos (\theta)^{-2}\right\rangle=E^{\text {free }}\left(\frac{2}{\pi}\right)^{2}=E^{\text {free }} / q_{\text {iso }} .
$$

Thus we expect that our method underestimates the azimuthal (non-potential) field component $B_{\varphi}$ by a factor of $(\pi / 2) \approx 1.6$ in the average, and thus the free energy by a factor of $(\pi / 2)^{2} \approx 2.5$. This bias can approximately be corrected with the isotropic twist correction factor $q_{i s o}=(\pi / 2)^{2} \approx 2.5$ in the determination of the free energy, i.e., $E^{\text {free }}=E_{\perp}^{\text {free }} q_{i s o}$. 


\section{NUMERIC CODE}

The Coronal Nonlinear Force-Free Field (COR-NLFFF) forward-fitting numerical code has evolved from an earlier version (Aschwanden and Malanushenko 2013) to the present version, achieving now a higher degree of accuracy in the measurement of the nonpotential magnetic energy $E_{N}$ and the free energy $E_{\text {free }}=E_{N}-E_{P}$. A flow chart of the latest version is provided in Fig. 2, which can be broken down into three major tasks: (1) the processing of the magnetic data; (2) the processing of the EUV data (including automated loop tracing), and (3) the forward-fitting of the analytical NLFFF approximation. In the following we briefly describe the numerical methods that are employed in the COR-NLFFF code, while a parametric study to establish the optimum choice of control parameters (Table 1) is documented in Appendix A.

\subsection{The Magnetic Field Decomposition}

The input of magnetic data is a line-of-sight magnetogram $B_{z}(x, y)$, in this case from HMI (Scherrer et al. 2012; Hoeksema et al. 2014) onboard the SDO spacecraft. We use the 45 s HMI data, which have been processed from $135 \mathrm{~s}$ time intervals. An HMI image covers the full-Sun and has a pixel size of $0.5^{\prime \prime}$, or approximately $\Delta x=0.00052$ solar radii. We are not using any photospheric vector magnetograph data, in contrast to the traditional NLFFF codes, because we consider the transverse components $B_{x}(x, y)$ and $B_{y}(x, y)$ as unreliable for coronal field extrapolations due to the non-forcefreeness of the photosphere (Fig. 1), and thus we keep them as free parameters, which will be computed from the constraints of the geometric shapes of observed coronal loops. We use only the line-of-sight magnetic field component $B_{z}(x, y)$ of the HMI data. The magnetic control parameters of the COR-NLFFF code are: the spatial resolution $\left(\Delta x_{m a g}\right)$ of (rebinned) magnetogram data used in the decomposition of (gaussian-like) point sources, and the number of decomposed magnetic source components $\left(n_{\text {mag }}\right)$. The numerical algorithm for magnetogram decomposition is described in Aschwanden (2010) and Aschwanden et al. (2012, Appendix A therein). A modification in the newest version of the code is the fitting of a 2D Gaussian function to local maxima of the magnetograms, which are iteraticely subtracted and the residuals within the full width at half mean (FWHM) are set to zero, in order to minimize the number of magnetic source components in the model. In addition, we normalize the fitted Gaussian to the same magnetic field strength as the local peak in the magnetogram, in order to conserve the magnetic flux $\left(\Phi=B \times \mathrm{FWHM}^{2}\right)$ and magnetic energy $\left(E_{B} \propto B^{2}\right)$. Examples of the Gaussian decomposition of the magnetogram are given in the parameteric study described in Appendix A (see also Figure 17).

\subsection{The Selection of Loops}

The second major task deals with the extraction of coronal loop coordinates from the EUV images. The optimization of the automated loop tracing code is described in a recent study (Aschwanden et al. 2013b), which is controlled by the lowpass filter constant $\left(n_{s m 1}\right)$ (boxcar pixels), the highpass filter $\left(n_{s m 2}=n_{s m 1}+2\right)$, the minimum loop curvature radius $\left(r_{\min }\right)$, the minimum loop length $\left(l_{\min }\right)$, and the loop length increment $(\Delta s)$, which all have been optimized (Appendix A) and the default values are given in Table 1.

A unique capability of our COR-NLFFF code is the synthesized processing of multiple EUV images in different wavelength filters, which encompsasses six coronal (94, 131, 171, 193, 211, $335 \AA$ ) and one chromospheric $(304 \AA)$ filter from AIA/SDO. How do we optimize the selection of loops in the forwardfitting code? We set up a number of control parameters that include the selected wavlengths $(\lambda)$, the 
maximum number $\left(n_{\text {loop }}\right)$ of loops extracted per wavelength filter, the minimum loop length $\left(l_{\text {min }}\right)$, and the minimum curvature radius $\left(r_{\min }\right)$ in the loop selection. We find that selections with an over-proportional contribution of large loops tends to favor potential field solutions (and thus a small ratio of the non-potential energy $q_{E}=E_{N} / E_{P}$, while a selection with a dominant contribution of small loops produces the opposite. Since large loops are found at relatively cool temperatures ( $T \approx 1-2 \mathrm{MK}$; i.e., 171 and $193 \AA$ ), while smaller loops in the core of active regions are found at hotter temperatures $(T \approx 10-20$ MK; i.e., 94 and $335 \AA)$, the choice of wavelengths $(\lambda)$ and the limit $\left(n_{\text {loop }}\right)$ on the extracted loops per wavelength filter play a decisive role. It is crucial to have a balanced amount of small and large loops in order to obtain a representative value for the free energy. A parametric study of varying the selection parameters $\left(\lambda, n_{\text {loop }}, l_{\text {min }}, r_{\min }\right)$ is given in Appendix A.

We implemented also criteria to automatically reject false loop structures, i.e., curvi-linear features that are caused by CCD pixel bleeding, CCD saturation, and diffraction patterns from the EUV entrance filter.

\subsection{Forward-Fitting Control Parameters}

The forward-fitting algorithm consists of the task of optimizing the free parameters $\alpha_{j}, j=1, \ldots, N_{m}$, associated with each magnetic charge in such a way that the 2D misalignment angle $\mu_{2}$ between an observed loop segment and a theoretical field line calculated at the same location is minimized. Forward-fitting with a large number of free parameters requires a customized numerical scheme that makes a reasonable trade-off between numerical accuracy and computational efficiency. A first numerical code that fits the analytical approximation (Equations 4-9) to a given analytical 3D magnetic field is described and tested in Aschwanden and Malanushenko (2013), forward-fitting to real solar data in 2D and 3D is conducted in Aschwanden (2013c) and computations of the free energies in Aschwanden (2013b). The multi-parameter optimization scheme used in the original code (Aschwanden and Malanushenko 2013) employed a hierarchical subidivision of $\alpha$-zones in the magnetic map that refines iteratively the $\alpha$-values into progressively smaller regions. In the latest version we use all magnetic source components in the forward-fitting of force-free $\alpha_{j}$ parameters. In addition we tested different optimization algorithms: (1) the Powell optimization scheme (which seeks the local minima of the parameters $\alpha_{j}$ sequentially during each iteration cycle), and (2) a gradient optimization scheme (which determines the gradient $d \mu_{j} / d \alpha_{j}$ of the misalignment angle $\mu_{j}$ as a function of the free parameter $\alpha_{j}$ during each iteration cycle. The Powell optimization generally converges to a smaller misaligment angle and is the method of choice in the COR-NLFFF code.

The mean misalignment angle $\mu_{2}$, which is minimized in the forward-fitting method, is defined by the root-mean-square (r.m.s.),

$$
\left\langle\mu_{2}\right\rangle=\sqrt{\frac{\sum_{i} \mu_{i}^{2}}{n_{\text {loop }}}}, \quad i=1, \ldots, n_{\text {loop }},
$$

where $\mu_{i}$ is the misalignment angle of loop $i$, which itself is the median of $n_{\text {seg }}$ loop segments (typically $n_{\text {segm }}=7$ ),

$$
\mu_{i}=\operatorname{median}\left(\mu_{i, k}\right), \quad k=1, \ldots, n_{\text {segm }} .
$$

Thus, the forward-fitting essentially consists of fitting the $\alpha$-values $\left(\alpha_{j}, j=1, \ldots, n_{f i t}\right)$ to the loop misalignment angles $\mu_{i, k}$, with $i=1, \ldots, n_{\text {loop }}$ and $k=1, \ldots, n_{\text {segm }}$. The optimization of the forward-fitting control parameters $\left(n_{\text {seg }}, \Delta \alpha_{j}, h_{\text {max }}, n_{\text {iter }}\right)$ is quantified in Appendix A.

There is also the problem of the missing third dimension in the observations. The forward-fitting algorithm minimizes the misalignment angle $\mu_{2}$ between an observed $2 \mathrm{D}$ loop coordinate $\left(x_{i}, y_{i}\right), i=0, \ldots, n_{f i t}$ 
and a theoretical 3D field line intersecting at $\left(x_{i}, y_{i}, z_{i}\right)$. The line-of-sight coordinate $z_{i}$ is not known a priori, unless we would employ stereoscopy (Aschwanden 2013c), but is a required quantity to select the proper theoretical field line. One constraint that we can use is the valid range of $z_{i}=\left[\left(1+h_{i}\right)^{2}-x_{i}^{2}-y_{i}^{2}\right]^{1 / 2}$ within a solution space corresponding to an altitude range of $0<h_{i}<h_{\max }$ at each position $\left(x_{i}, y_{i}\right)$. We assume that each observed loop segment can be approximated with a circular segment as a function of the LOS-coordinate $z_{i}$,

$$
z_{i, k}(s)=z_{i, 0}+r_{\text {curv }} \sin \left[\varphi_{1}+\left(\varphi_{2}-\varphi_{1}\right) q_{\text {seg }, k}\right],
$$

where the starting angle $\varphi_{1}$ of the circular segment can be anywhere within the range of $0^{\circ} \leq \varphi_{1} \leq+180^{\circ}$, while the end angle $\varphi_{2}$ can be anywhere within the range of $\varphi_{1} \leq \varphi_{2} \leq+180^{\circ}$, where $0<q_{\text {seg }}<1$ is a normalized loop length coordinate, $r_{c u r v}$ is the curvature radius of the circular segment, and $z_{0}$ is the center height of the circular segment, adjusted in such a way that the circular segment is always located in the altitude range of the computation box with $h=\left[0, h_{\max }\right]$. We choose $n_{c}=n_{\text {seg }}=7$ starting angles $\varphi_{1}$ (each $30^{0}$ apart) and $n_{h}=n_{\text {seg }}=7$ possible height ranges, which yields $n=n_{h} \times n_{c}\left(n_{c}-1\right) / 2=147$ different trial geometric shapes to estimate the heights $z_{i, k}$ in the $k=1, \ldots, n_{\text {seg }}$ loop segments of each loop $i$.

After every iteration cycle of forward-fitting a set of $\alpha_{j}$-values, we compute the trial geometric shapes (Equation 15) with the new $\alpha_{j}$ values and adjust the heights $z_{i}^{\text {trial }}$ at the midpoints of each loop, by

minimizing the misaligment angles $\mu_{2}$ between the loop coordinates $\left(x_{i}, y_{i}, z_{i}^{\text {trial }}\right)$ and the theoretical field lines $\left(x_{i}, y_{i}, z_{i}^{\text {model }}\right)$. In this way we optimize both the force-free parameters $\alpha_{j}$ and the 3D-coordinate $z_{i}$ of the loop midpoints. Alternative trial geometries used for magnetic modeling of 3D loops are Bezier functions (Gary et al. 2014a,b).

\section{OBSERVATIONS AND DATA ANALYSIS}

SDO data are most suitable for our type of magnetic modeling of active regions, because HMI and AIA provide both high-resolution magnetograms and simultaneous multi-wavelength EUV images. An additional requirement for a suitable active region is a location near Sun disk center and the appearance of many (possibly twisted) coronal loops. Some of such active regions have already been modeled with NLFFF codes that allow us to compare results. The active region NOAA 11158 produced one of the most spectacular flares (GOES X2.2 class) during the first two years of the SDO mission and has been intensively studied since then, documented in over 40 publications so far (e.g., Sun et al. 2012a; Schrijver et al. 2011; Wang et al. 2012; Kosovichev 2011, Aschwanden et al. 2013a). See Section 5.1 for more details and references.

\subsection{AIA and HMI Observations}

We study the evolution of active region 11158 over 5 days, from 2011 February 12, 00 UT, to February 17, $00 \mathrm{UT}$, which is the same interval as analyzed in Sun et al. (2012a). A sample set of 7 images, observed with AIA/SDO (Lemen et al. 2012; Boerner et al. 2012) on 2011 February 15, 00:00:00 UT (about two hours before the GOES X2.2-class flare), is shown in Fig. 3 with the wavelengths of 94, 131, 171, 193, 211, 304, and $335 \AA$. A near-simultaneous magnetogram observed with HMI/SDO within one minute (2012 February $14,23: 58: 57 \mathrm{UT}$ ) is shown in Fig. 3 also. The pixel size of AIA is $0.6^{\prime \prime}$ (with a spatial resolution of $\approx 2.5$ pixels), and the pixel size of HMI is 0.5". These 8 images shown in Fig. 3 represent all data input that is needed for our magnetic NLFFF modeling. No vector magnetograph data from HMI are used, because our method needs only the line-of-sight component and treats the transverse magnetic field components as free 
parameters.

We show the loop structures enhanced in form of bandpass-filtered images in Fig. 4. The bandbass used here consists of a lowpass filter with a boxcar length of $n s m_{1}=5$ pixels, and a highpass filter with a boxcar length of $n s m_{2}=7$ pixels. Thus it enhances most efficiently loops with a cross-sectional width in the range of 5-7 pixels, which corresponds to a cross-sectional width of $w \approx 2-3 \mathrm{Mm}$. Obviously we see coronal loops in all wavelengths (Fig. 4), but filters with cool coronal temperatures (171, 193, $211 \AA ; T_{e} \approx 1-2 \mathrm{MK}$ ) show more large-scale loops that overarch the active region, while filters sensitive to hotter temperatures $(94,131$ $\AA)$ reveal more highly twisted structures in the core of the active region, similar to the structures seen at chromospheric temperatures $(304 \AA ; T \approx 0.05 \mathrm{MK})$. Loop structures in this active region were analyzed in almost the same set of images two hours before the X-class flare, and have been subjected to automated temperature and emission measure analysis and automated loop tracing (Aschwanden et al. 2013a).

\subsection{Automated Loop Tracing}

The automated loop tracing in this active region is shown separately for each temperature filter in Fig 5, conducted with an improved algorithm (Aschwanden et al. 2013a) than used previously. For the particular run shown in Fig. 5 we used the following control parameters (defined in Aschwanden et al. 2012): a maximum number of $n_{L}=50$ loops per wavelength filter, a minimum curvature radius of $r_{\text {min }}=30$ pixels, a minimum loop length of $l_{\min }=30$ pixels, a lowpass filter of $n_{s m 1}=5$ pixels, a highpass of $n_{s m 2}=7$ pixels, and a flux threshold level that corresponds to $[5,2,0,0,1,5,5]$ times the noise level (median) for the wavelengths $[94,131,171,193,211,304,335] \mathrm{A}$, evaluated in stripes at each of the four image boundaries. With this particular setup the code picked up a total of $n=238$ curvi-linear loop structures (Fig. 5, bottom right), synthesized from all wavelengths, from a minimum of $n=89$ structures in the $94 \AA$ filter, and a maximum of $n=149$ structures in the $193 \AA$ filter. The automated tracing reveals a large number of bipolar loops and peripheral (open-field) fan structures in the 171, 193, and 211 filters, while the other filters exhibit shorter structures in the core of the active region surrounding the flare site. The complementary nature of loop tracing is most conspicuous between 171 and $335 \AA$ (Fig. 5).

\subsection{Nonlinear Force-Free Field Computation}

The results of the forward-fitting of the analytical NLFFF approximation is shown for each wavelength separately in Fig. 6. The panels in Fig. 6 show the automatically traced 2D coordinates of the loop segments (blue curves) and their midpoints (blue diamonds), where an intersecting fieldline segment of the NLFFF best-fit model with equal length is indicated (red curve), as well as the full field line (orange curve), starting and ending either at the solar surface or at a boundary side of the computation box, which extends over the field-of-view shown in the Figures 3-6 and a vertical height of $h_{\max }=0.15$ solar radii above the solar surface. The control parameter of these fits are: $n_{m a g}=100$ magnetic sources; a spatial resolution corresponding to one AIA pixel size; a maximum range of the force-free parameter $\Delta \alpha_{\max }= \pm 10 R_{\odot}^{-1}$ per iteration step; a maximum number of $n_{\text {loop }}=50$ loops per wavelength filter; and a minimum length of $l_{\text {min }}=30$ pixels for the selected loops. A simultaneous fit to a subset of 238 loops synthsized from the 50 brightest ones in each filter is shown in Fig. 6 (bottom right panel).

It is interesting to compare the best-fit NLFFF solutions in each wavelength, because they reveal the model sensitivity to the loop selection. The wavelengths with the largest number of loops are 171, 193, and 
$211 \AA$, amounting to $n_{\text {loop }} \approx 110-150$. The other wavelengths, however, have a smaller number of loops $\left(n_{\text {Loop }} \approx 10-70\right)$, have smaller loop lengths, are preferentially located in the core of the active region, and have lower altitudes. It appears that these two groups of loops have also different degrees of non-potentiality: the large-scale loops over-arching the active region are close to the potential field solution with low $\alpha$-values, while the small-scale loops in the core of the active region are stronger twisted, including the sigmoid-like filament in the center, and have a higher degree of non-potentiality, with higher $\alpha$-values.

The relaxation of a twisted non-potential field to a more dipolar potential field can also be seen visually by inspecting two of the COR-NLFFF solutions for AR 11158 before the X2.2 flare (Fig. 7, top panel: 2011Feb-15, 01:48 UT), and shortly after the flare peak (Fig. 7, bottom panel: 2011-Feb-15, 02:06 UT). The highly twisted central filament in the northern pair of sunspots of the quadrupolar active region relaxes from a helical twist of about one turn to an almost dipolar geometry with little twist. A movie of the magnetic field caclulated with COR-NLFFF during a time interval of 6 hours is contained in the supplementary electronic material to this paper.

\subsection{Time Evolution of the Free Energy}

We study now the time evolution of the potential magnetic energy $E_{P}(t)$, the non-potential magnetic energy $E_{N}(t)$, and the free magnetic energy or difference $E_{\text {free }}(t)=E_{N}(t)-E_{P}(t)$ for AR 11158 . These energies are integrated over a computation box that is aligned with the spherical solar surface, with a fieldof-view of $0.3 \times 0.3$ solar radii, and with an altitude range of $h_{\max }=0.15$ solar radii, centered at heliographic coordinates $[l(t), b(t)]$ following the solar rotation. The central meridian passage of AR 11158 was at 2011 Feb 14, $00 \mathrm{UT}$, and the heliographic latitude is $b(t) \approx-15^{\circ}$.

The evolution of the potential magnetic energy starts with a low amount of $E_{P} \approx 1 \times 10^{32}$ erg on 2011-Febr-12, and then ramps up almost monotonically to a value of $E_{P} \approx 8 \times 10^{32}$ erg during the next two days (Fig. 8b), so the total magnetic energy increases by a factor of about 8 during the 2 days before the X2.2 flare on 2011-Feb-15. Both our COR-NLFFF code and the Wiegelmann NLFFF code (Sun et al. 2012a) agree well (within a few percents) in the total potential energy during the first 3 days, but deviate by about $30 \%$ during the last two days, when the active region was $\gtrsim 25^{\circ}$ away from the centeral meridian, perhaps due to some side effect of the de-rotation projection to disk center applied in Sun et al. (2012a), or due to center-of-limb effects of the LOS-component decomposition in our COR-NLFFF code.

The time evolution of the nonpotential magnetic energy $E_{N}(t)$, obtained from $2 \times 1200$ forward-fits with the COR-NLFFF code to traced coronal loops, with a 6-min cadence, is shown in Fig. 8c (blue profile), and is compared with the Wiegelmann NLFFF code with 12-min intervals (red curves), calculated previously also wtih 1-hour intervals (orange curves in Fig. 8; see also Fig. 4 in Sun et al. 2012a).

We show the evolution of the free energy $E_{\text {free }}(t)=E_{N}(t)-E_{P}(t)$ in Fig. (8d). There is a rapid increase in the free energy at the beginning of the second day (2011-Feb-13) as calculated with the Wiegelmann code, while no such jump is evident as caclulated from the HMI line-of-sight component with the COR-NLFFF code, which is interpreted as an episode of rapid magentic flux emergence with strong horizontal field and little vertical field, occurring in the north-western bipole of the active region. Apparently there are no bright loops associated with the emerging field, because we detect no increase in free energy based on the coronal NLFFF code.

Since the free energy is a small quantity obtained from the difference of two large quantities, the relative 
uncertainties are larger than the relative uncertainties of the absolute energies. We do not know a priori which code produces more reliable values of the free energy, since each code has its own caveats and strengths, and the two codes are using complementary (photospheric versus coronal) information. We find the following differences in the results of the free energy obtained with the Wiegelmann NLFFF code, which uses preprocessed photospheric vector data (Sun et al. 2012a), and our COR-NLFFF code, based on forward-fitting to (automatically) traced coronal loops: (1) The absolute values of the free energies differ up to a factor of 4 between the two codes; (2) The Wiegelmann NLFFF code yields a relatively smooth evolution of the magnetic energy, while our COR-NLFFF reveals more rapid fluctuations in the time evolution of the free energy (Fig. 8d); (3) There are a number of GOES flares where the COR-NLFFF code shows substantial changes in the free energy that appear to be correlated with the GOES fluxes, while the Wiegelmann NLFFF code shows only very small fluctuations of the free energy. We will investigate these differences in more detail in the following.

Let us have a look at some fitting parameters in order to see whether they could be responsible for the fluctuations of the obtained free energy. The number of automatically selected loops in the 1200 forwardfitting runs are shown in Fig. (8e), and has a mean of $n_{\text {loop }}=248 \pm 18$. The number of selected loops has a lowest value of $n_{\text {loop }}=192$ during the first day, when the GOES flux as well as the total magnetic energy is also low, which reflects the fact that less detectable loops existed during this day. In the overall, there is much less fluctuations in the number of loops $n_{\text {loop }}(t)$ than in the free energy $E_{\text {free }}(t)$ (Fig. 8d).

The goodness-of-fit test of the 1200 forward-fitting runs is given in terms of the median 2D misalignment angle $\mu_{2}(t)$ in Fig. (8f), with a mean of $\mu_{2}=4.4^{\circ} \pm 0.7^{\circ}$, which is to be compared with $\mu_{2} \approx 10^{\circ}$ for potential field models. The goodness-of-fit $\mu_{2}(t)$ is fairly uniform during the 5 days, even during flaring episodes, thanks to the new improvements in the COR-NLFFF code that eliminates false loop structures caused by CCD pixel bleeding, CCD saturation (for too long exposure times), and diffraction patterns due to the EUV entrance filters.

\subsection{Free Energy Changes and Correlations with GOES Flares}

We show an expanded time profile of the free energy $E_{\text {free }}(t)$ of AR 11158 in Fig. 9, along with the light curves of the GOES 1-8 A flux, which contains 36 flares above the GOES C0-class level during the 2011 Feb 12-17 period. Note that the free energy calculated with the COR-NLFFF code exhibits much stronger fluctuations than the PHOT-NLFFF (Wiegelmann) code in the later days, while both codes show an absence of significant fluctuations during the first day (2011 Feb 12).

We investigate now changes in the magnetic energy $\Delta E_{\text {free }}(t)$ specifically during these 36 flare time intervals. A sample of 9 flare time intervals are shown enlarged in Fig. 10. We determined the flare start time $\left(t_{\text {start }}\right)$, peak time $\left(t_{\text {peak }}\right)$, and end time $\left(t_{\text {end }}\right)$ of these 36 events (Table 2 ) based on standard NOAA flare catalog, where the end time is defined when the GOES flux drops to $50 \%$ of the peak flux. We define a decrease of the free energy as follows,

$$
\Delta E_{\text {free }}=\left(E_{\text {post }}-E_{\text {pre }}\right)=E_{\text {free }}\left(t=t_{\text {post }}\right)-E_{\text {free }}\left(t=t_{\text {pre }}\right),
$$

where the preflare reference time $t_{\text {pre }}$ is identified by the maximum of the free energy during the preflare time interval $\left[t_{\text {start }}-0.3 \mathrm{hr}, t_{\text {peak }}\right]$, and the postflare reference time $t_{\text {post }}$ is identified by the minimum of the free energy during the time interval $\left[t_{\text {start }}, t_{\text {peak }}+0.3 \mathrm{hr}\right]$. The so-determined time intervals $\left[t_{\text {pre }}, t_{\text {post }}\right]$ of maximum energy dissipation during the risetime of the flare (within a margin of $0.3 \mathrm{hr}$ ) are shown for both 
the COR-NLFFF code (blue curves in Fig. 10) and the Wiegelmann NLFFF code (red curves in Fig. 10). The uncertainties of the free energy, $E_{\text {free }} \pm \sigma_{E}$, are empirically determined from the median daily fluctuations of the free energy, which varied in the range of $\sigma_{E}=(2-34) \times 10^{30} \mathrm{erg}$ for the COR-NLFFF code, and $\sigma_{E}=(1.6-6) \times 10^{30} \mathrm{erg}$ for the PHOT-NLFFF code. The free energies and their uncertainties, as well as the energy decrease $\left(\Delta E_{\text {free }}\right)$ during the 36 flare events are listed in Table 2, for both the COR-NLFFF and the (Wiegelmann) PHOT-NLFFF code.

The main result is that 29 out of the 36 flare events calculated with our COR-NLFFF code exhibit a significant decrease in free energy during the flare time interval, while only 7 events show no significant change, either due to the smallness of the energy change or the large uncertainty of the method. Likewise we find a significant energy decrease for most of the flares with the Wiegelmann NLFFF code, but about a 3-10 times smaller amount of energy decreases. For instance, during the X2.2 flare on 2011-Feb-15, 02 UT (event \#16, Fig. 10 top right panel), we detect an energy decrease of $\Delta E_{\text {free }}=-(62 \pm 28) \times 10^{30} \mathrm{erg}$ with the COR-NLFFF code, while about the half amount of $\Delta E_{\text {free }}=-(37 \pm 4) \times 10^{30}$ erg was detected with the Wiegelmann NLFFF code (Sun et al. 2012a).

In Fig. 11 we compare the decreases of free energy $(-\Delta E)$ with the GOES 1-8 $\AA$ fluxes and find a weak trend of a correlation between the magnetic energy and the soft X-ray flux for the photospheric NLFFF method (Sun et al. 2012), with a linear regression fit of $\log (\Delta E) \propto \log \left(F_{G O E S}\right)^{0.26 \pm 0.06}$, while the coronal NLFFF method shows no significant correlation. In flare models with magnetic reconnection we would expect that the dissipated magnetic energy is correlated with the radiative output of heated plasma during flares. The scatterplot in Fig. 11 illustrates also that the energy decreases detected with the photospheric NLFFF code are about an order of magnitude smaller than those with the coronal NLFFF code for small flares, and a factor of three for the largest flares.

In Fig. 12 we show the correlation of potential energies (Fig. 12 left panel) and nonpotential energies (Fig. 12 right panel) between the coronal NLFFF code and the photospheric NLFFF code by Wiegelmann for 600 time steps (12-min cadence) during the entire 5-day period. Both the potential and nonpotential energies correlate well during the first 3 days (when the potential energy was $E_{P} \lesssim 10^{33} \mathrm{erg}$ ), but deviate during the last two days. At this time it is not clear whether this discrepancy is due to some systematic center-to-limb effect in the de-rotation of photospheric vector magnetograms (Sun et al. 2012a), or due to a center-to-limb effect in the decomposition of LOS magnetograms (Section 3.1). In principle, the coronal NLFFF code can represent vertically twisted field lines at any longitude, if the magnetic charges are properly decomposed from the magnetogram.

A linear regression fit in Fig. 12 shows that the potential energy is recovered by $85 \%$ with the coronal NLFFF code, while the nonpotential energy is recovered by $74 \%$. Since the magnetic energy scales with the square of the magnetic field strength, the potential field is recovered by $92 \%$, using $n_{\text {mag }}=100$ magnetic source components in our decomposition technique. This difference causes a reduction of the free energy up to a factor of 4 for the coronal NLFFF code, compared with the photospheric (Wiegelmann) NLFFF

code (Fig. 8d), which could indicate a systematic effect of higher twist in the non-forcefree zones of the photosphere and lower chromosphere.

\subsection{Loop Illumination Effects}

When we analyze the time evolution of the free energy $E_{\text {free }}(t)$ in the time profiles shown in Figs. 9 and 10 (blue histograms), we see that almost none of the flares exhibits the expected behavior of a near-constant 
high level of free energy before the flare, which then decreases to a lower level during the impulsive flare phase, so that a simple energy difference between the levels before and after the flare could be used as a measure of the dissipated magnetic energy. Instead we observe often a rapid increase of the free energy at the beginning of the impulsive flare phase, which we interpret as an illumination effect of highly twisted loop structures (sigmoids) that yield a high contribution of nonpotential energy to the free energy (time step $t_{1} \mapsto t_{2}$ in Fig. 13). In this scenario, it is still possible to measure the decrease of free energy after the peak (time step $t_{2} \mapsto t_{3}$ in Fig. 13), regardless whether the preflare level is low (for invisible sigmoids) or high (after illumination, say when the flare-associated chromospheric evaporation process fills the sigmoids with dense plasma. Once a sigmoid gets brighter, it will be detected with our automated loop detection scheme and will cause higher amounts of free energy in the COR-NLFFF forward-fitting algorithm.

In some cases we see multiple peaks of the free energy during the flare phase (e.g., secondary peak in time step $t_{3} \mapsto t_{4}$ in Fig. 13), which are likely to be associated with spatially different twisted structures. We can apply the same interpretation of an illumination effect during intervals of increasing free energy and magnetic dissipation during the time intervals of decreasing free energy (time step $t_{4} \mapsto t_{5}$ in Fig. 13). In order to caclulate the total dissipated energy, we would have to add each decrease of the free energy during the impulsive flare phase. If multiple such episodes overlap in time, even the sum of all free energy decreases will be only a lower limit to the total dissipated energy, because the time overlap will partially cancel energy increases (due to illumination effects) and energy decreases.

In summary, we conclude that our method of estimating the dissipated free energy from the difference between the peak of the free energy during the preflare phase and the minimum after the impulsive flare phase will generally yield a lower limit. This scenario may be tested in future work by measuring the illumination effects and dissipation of free energy in spatially separated regions and with higher time resolution, and this way may yield more accurate estimates of the total dissipated magnetic energy in flares.

\section{DISCUSSION}

\subsection{Previous Studies}

The magnetic field of AR 11158 during a few days around the X2.2 flare on 2011-Feb-15, 02 UT, has been the subject of at least 40 different publications for the following reasons: (1) The X2.2 flare was the first X-class flare observed with SDO; (2) the active region has been observed near the central meridian, which is favorable for any magnetic field extrapolation method, and (3) the HMI/SDO data have been calibrated and released (Hoeksema et al. 2014).

\subsubsection{Previous Studies on the X2.2 flare}

The over 40 publications of this active region and its main X2.2 flare cover: the magnetic evolution as computed with nonlinear force-free codes (Sun et al. 2012a; Jiang and Feng 2013; Inoue et al. 2013; Tarr et al. 2013) and with stereoscopic comparisons (Wang et al. 2014), MHD magnetic flux-rope modeling (Schrijver et al. 2011), the photospheric magnetic response to the flare (Wang et al. 2012, 2013; Liu et al. 2012, 2013; Petrie 2012a,b), magnetic field collapse and quasi-periodic loop oscillations during the flare

(Gosain 2012; Dolla et al. 2012), coronal waves (Schrijver et al. 2011; Olmedo et al. 2012), coronal mass ejection and interplanetary kinematics (Schrijver et al. 2011; Maricic et al. 2013a, 2013b), velocity and 
magnetic transients of the flare (Maurya et al. 2012; Vemareddy et al. 2012a,b; Petrie 2012a,b), horizontal flow motion during the flare (Beauregard et al. 2012; Liu et al. 2013), the sunquakes and helioseismic response to the flare (Kosovichev 2011; Zharkov et al. 2011, 2013; Alvarado-Gomez et al. 2012), rapid sunspot rotation during the flare (Jiang et al. 2012; Vemareddy et al. 2012a,b), the EUV continuum evolution during the flare (Milligan et al. 2012), high-speed outflows shortly before the flare (Su et al. 2012), flare-associated radio bursts (Borovik et al. 2012; Chen et al. 2013; Yashiro et al. 2014), magnetic modeling and flare trigger of a preceding M-class flare (Kusano et al. 2012; Toriumi et al. 2013), and spectroscopy of flows in the kernel of a following M-class flare (Young et al. 2013).

\subsubsection{Previous Studies on Active Region NOAA 11158}

Studies on AR 11158, which hosted the X2.2 flare, include the evolution of the nonpotentiality and helicity of the active region (Liu and Schuck 2012; Jing et al. 2012; Song et al. 2013; Inoue et al. 2013; Tziotziou et al. 2013; Vemareddy 2012a,b), the quadrupolar magnetic configuration of the active region (Sun et al. 2012b), the magnetic energy distribution of the active region (Shen et al. 2013), the magnetic calibration of photospheric Doppler velocities in the active region (Welsch et al. 2013), automated loop tracing, and emission measure and temperature analysis of the active region (Aschwanden et al. 2013a).

\subsection{Energy Budget of the X2.2 Flare on 2011-Feb-15}

A primary goal of this study is to establish the amount of energy that is dissipated during solar flares, which we hope to derive from the change of free magnetic energy during the flare time intervals. The largest flare during the analyzed time interval, the X2.2 flare on 2011 February 15, 02 UT, is the most suitable event in this context, since we have free energy calculations from multiple NLFFF codes: the Wiegelmann code (Sun et al. 2012a), an MHD relaxation code (Jiang and Feng 2013), and our COR-NLFFF code based on forward-fitting to coronal loops. In Table 3 we compile the different forms of energies that have been calculated for this flare.

The total (nonpotential) magnetic energy averaged from the four values listed in Table 3 has a mean of $E_{N}=(10.4 \pm 1.4) \times 10^{32} \mathrm{erg}$, which corresponds to a mutual agreement within $\approx 15 \%$. The potential energies have a mean of $E_{P}=(8.7 \pm 0.8) \times 10^{32} \mathrm{erg}$ and agree within $\approx 10 \%$. The free magnetic energy has a somewhat larger scatter, within a range of $E_{\text {free }}=(1.65-2.4) \times 10^{32} \mathrm{erg}$ for the standard NLFFF codes, with or without pre-processing, while we find with the COR-NLFFF code a similar value, i.e., $E_{\text {free }}=(0.98 \pm 0.20) \times 10^{32}$ erg. However, the largest discrepancy is found for the decrease of free energy during the X2.2-class flare, for which a relatively low value of $\Delta E=E_{\text {post }}-E_{\text {pre }}=(0.37 \pm 0.04) \times 10^{32}$ erg has been found with the Wiegelmann NLFFF code (Sun et al. 2012a), a mere $\approx 15 \%$ of the available free energy, while we find about a double value for the energy decrease of $\Delta E=E_{\text {post }}-E_{\text {pre }}=(0.62 \pm 0.28) \times 10^{32}$ erg with our coronal forward-fitting code, which makes up about $60 \%$ of the free available energy. This is a very intriguing result, because it sets a lower limit on the energy budget of the flare and may invalidate the results of some NLFFF codes.

We have some other complementary information on the energy input of this flare, from minimum-current corona modeling and from the virial theorem. Using a magnetic charge topology (similar to the magnetic source decomposition in our COR-NLFFF code, see Sections 2.2 and 3.1), together with the minimum current corona model, the amount of reconnecting flux was calculated and a drop in the free magnetic energy of 
$\Delta E_{M C C}=-1.68 \times 10^{32} \mathrm{erg}$ was found during the X2.2 flare (Tarr et al. 2013), which agrees within a factor of about two with our COR-NLFFF code and the Wiegelmann NLFFF code (Sun et al. 2012a). The nonpotentiality can also be calculated with the virial theorem (Chandrasekhar 1961; Low 1982), which yields an energy drop of $\Delta E_{\text {virial }}=(1.05 \pm 1.04) \times 10^{32} \mathrm{erg}$ (Vemareddy et al. 2012b), and a similar amount of $\Delta E_{\text {virial }}=0.84 \times 10^{32} \mathrm{erg}$ from an independent other calculation (Tziotziou et al. 2013). Both of these values obtained with the virial theorem are comparable with our forward-fitting method, but exceed the value obtained with the Wiegelmann code (using pre-processed data) by a factor of $\approx 3$ (Sun et al. 2012a).

Another test of the plausibility of a free energy calculation is the positive balance after energy losses during a flare. The major part of the dissipated energy in flares is believed to be converted into acceleration of nonthermal particles and heating of the chromosphere by precipitating particles (ions and electrons). A calculation of the nonthermal energy spectrum in the range of $E=25-50 \mathrm{keV}$ using RHESSI data yielded a value of $E_{H X R}=0.54 \times 10^{32} \mathrm{erg}$ (Sun et al. 2012a), which exceeds the energy budget caclulated with the Wiegelmann code $\left(\Delta E=(0.37 \pm 0.04) \times 10^{32} \mathrm{erg}\right)$, while it dissipates less than the magnetic energy budget calculated with our forward-fitting code $\left(\Delta E=(0.62 \pm 0.28) \times 10^{32} \mathrm{erg}\right)$.

On the other hand, the total thermal energy produced in a flare can also be estimated from the differential emission measure (DEM) distribution obtained in soft and EUV wavelengths, for which we find at the flare peak, using AIA/SDO data, a total thermal energy of $E_{t h}=0.2 \times 10^{32}$ erg (which makes about $30 \%$ of the energy budget), with a peak temperature of $T_{p}=17.8 \mathrm{MK}$, a peak electron density of $n_{p}=3.2 \times 10^{11}$ $\mathrm{cm}^{-3}$ (assuming a filling factor of unity), and a flare radius of $L_{p}=16.3 \mathrm{Mm}$ (Aschwanden et al. 2013a). Other forms of energy losses have been determined in Ly $\alpha$, soft X-rays, Ly continuum, etc. (Table 3) with EVE/SDO (Milligan et al. 2012), which are all smaller than $\approx 1 \%$ of the free magnetic energy.

In summary, the decrease in free energy during the X2.2 flare is consistently determined to be $\Delta E \approx$ $(0.6-1.0) \times 10^{30} \mathrm{erg}$ with our forward-fitting code and with the virial theorem. The estimated energy losses due to acceleration of nonthermal particles and heating of the thermal plasma do not exceed this energy budget. The same conclusion holds for the 5 M-class flares that occurred in the same active region during the time period of 2011 Febr 12 to 17, for which we have energy changes calculated with the virial theorem (Vemareddy et al. 2012b; Tziotziou et al. 2013), and for which we have calculations of the total thermal energy (Aschwanden et al. 2013a), as compiled in Table 4. For additional comparisons of the energy budget in flares see also Fig. 4 in Emslie et al. (2012), and Emslie et al. (2004, 2013). In the latter studies, however, the free magnetic energy is not determined, but only the total potential field energy is given instead, which is about an order of magnitude larger than the free energy that is available for energy dissipation during flares. Therefore, our study provides much stronger contraints on the upper limit of the flare energy budget.

\subsection{Photospheric versus Coronal Magnetic Field Constraints}

This is the first study that quantitatively compares the coronal magnetic field computed by photospheric (extrapolation) and by coronal (forward-fitting) methods. In principle we aim to calculate the same nonlinear force-free field solution in a given computation box, but the significant differences we find here may provide important information on systematic errors of each magnetic field calculation method, or on the non-forcefreeness in the lower chromosphere. A recent review on the success and future improvements of NLFFF codes is given in Regnier (2013). 


\subsubsection{Comparison and Discrepancies}

Here we discuss a quantitative comparison between the photospheric NLFFF extrapolation code (Wiegelmann (2004), as applied in Sun et al. (2012a), and the coronal forward-fitting (COR-NLFFF) code used in this study. In Fig. 8 we show the evolution of the free energy over a time interval of 5 days computed in 1200 time steps (Fig. 8d), along with the potential (Fig. 8b) and nonpotential energy energy (Fig. 8c). We show also the correlation of the potential $E_{P}$ and nonpotential energy $E_{N}$ between the two codes in Fig. 12. We make the following findings: (1) The potential magnetic energy agrees with good accuracy between the two codes, at least during the first three days when the active region is near disk center. It is not clear whether the deviations during the last two days is due to a center-to-limb effect or due to a difference in the computation box. (2) The free energy obtained with the Wiegelmann NLFFF code exceeds that of the COR-NLFFF code up to a factor of 4 (Fig. 8d); (3) The decrease of the free energy measured with the Wiegelmann NLFFF code during flares is reduced by an amount of up to an order of magnitude, compared with the COR-NLFFF code. The energy descrease falls short of the nonthermal energy required to accelerate hard X-ray producing electrons during the X2.2 flare. On the other side, the free energy decrease during the X2.2 flare obtained with the COR-NLFFF code, $\Delta E_{\text {free }} \approx 0.6 \times 10^{32} \mathrm{erg}$, is consistent with the values obtained with the virial theorem (Vemareddy et al. 2012; Tziotziou et al. 2013). (4) The time profile of the free energy obtained with the Wiegelmann code exhibits about an order of magnitude less variability than with the COR-NLFFF code. In the following we discuss different effects that possibly could explain these discrepancies between the two codes.

\subsubsection{Spatial Resolution}

Both codes are using the original HMI data with a pixel size of $0.5^{\prime \prime}$. The HMI data used for the Wiegelmann NLFFF code are averaged down to two pixels (1.0") (Sun et al. 2012a), while the data used for the COR-NLFFF code are rebinned to three HMI pixels $\left(1.5^{\prime \prime}\right)$, for which the optimum match in the Gaussian decomposition of the line-of-sight magnetograms was established (Fig. 15a). It was speculated (Sun et al. 2012a) whether small-scale fields with large gradients could cause electric currents that are unresolved with the averaged HMI data, which could lower the resulting free energy. However, the spatial resolution of HMI data should affect both codes in a similar manner, since both codes used averaging of two or three pixels, and thus the averaging cannot explain the discrepancy of the obtained free energy between the two codes.

\subsubsection{Computation Box}

One constraint of standard NLFFF codes is the planar computation box with cartesian geometry, which requires a remapping of an active region at heliographic position $(l, b)$ to disk center $(0,0)$. The remapping transforms a fraction of the transverse field to the line-of-sight field component, which scales with the sine-function of the center-to-limb distance. In the study of Sun et al. (2012a), re-mapping of the HMI magnetogram using the Lambert equal area projection has been used, which is different from the COR-NLFFF code, where the full sphericity of the Sun is taken into account and no remapping is needed. However, since the X2.2 flare occurred near disk center, this effect should be very small and cannot explain the discrepancy in the free energy. 


\subsubsection{Time resolution}

For the forward-fitting to coronal loops we used the HMI $45 \mathrm{~s}$ data, which were processed by the HMI team from $135 \mathrm{~s}$ time intervals, so we see variability of the magnetic field down to about 2 minutes. The time step in modeling of AIA data is 6 minutes, but the exposure times of AIA are typically $\approx 2 \mathrm{~s}$. We ran the Wiegelmann NLFFF code with a time step of 12 minutes, which was processed by the HMI team from $1350 \mathrm{~s}$ (22.5 minute) time intervals. Thus part of the much lower modulation depth of the free energy calculated with the Wiegelmann NLFFF code could be due to the poorer time resolution. The data noise in the HMI data with 1350 s would be, compared with the HMI 135 s data used for coronal forward-fitting, a

factor of $\sqrt{10} \approx 3.3$ lower. However, since we detect about a factor of 10 less decrease of free energy during flares between the two methods (Fig. 11), there is still a factor of 3 less modulation depth unexplained.

\subsubsection{Pre-Processing of HMI Data}

Before using the Wiegelmann NLFFF code, the photospheric vector magnetograph data were "preprocessed", a procedure that minimizes the flux, force, and torque of the 3D magnetic field vectors (Wiegelmann et al. 2006). It is suspected that the pro-processing may introduce too much smoothing (or time averaging) in the photospheric data, which could reduce the electric currents and this way causes an underestimate of the free energy (Sun et al. 2012a). In the study of Jiang and Feng (2013), the pre-processed data yield about 10\% smaller total and potential energies than the raw data, resulting into about $15 \%$ less free energy for the X2.2 flare. This small difference cannot explain why we measure a factor of 2 different decrease in the free energy during the X2.2 flare.

\subsubsection{Coronal Loop Geometry}

The COR-NLFFF code performs forward-fitting of an approximate NLFFF solution to the shapes of coronal loops. This code is designed to fit the observed loop geometries, and thus retrieves the helical twist of loops. The helical twist of magnetic field lines plays a key role before large flares, such as observed here, which was confirmed with a 3D NLFFF magnetohydrodynamic (MHD) relaxation method (Inoue et al. 2013). The latter method determined one-half to one-full twist before the M6.6 and the X2.2 flare (Inoue et al. 2013), which clearly untwisted after the X2.2 flare, as it can be seen in the northern portion of AR 11158 in Fig. 7, depicted in two forward-fits before and after the flare peak.

The accuracy of the best-fit solutions of the COR-NLFFF code is typically a median misalignment angle of $\mu_{2} \approx 4.5^{\circ}$ (Fig. 8 bottom), which corresponds to a 3D misalignment angle of $\mu_{3}=\mu_{2} \sqrt{(3 / 2)} \approx 5.5^{\circ}$. In contrast, standard NLFFF codes, which by definition do not use any information of the observed coronal geometry, end up with typical 3D misalignment angles in the range of $\mu_{3} \approx 24^{\circ}-40^{\circ}$ (DeRosa et al. 2009). The misalignment angle $\mu_{3}$ is directly related to the force-free parameter $\alpha$, and thus to the free energy. If a misalignment angle $\mu$ increases to the double value in a bad fit, this translates into a doubled azimuthal (nonpotential) magnetic field component $B_{\varphi} \propto B_{\text {pot }} \tan (\mu)$, an thus to a free energy ratio squared, i.e., $q_{\text {free }}=\left(B_{\varphi} / B_{\text {pot }}\right)^{2}=\tan (\mu)^{2}$. For instance, if a NLFFF solution is misaligned by $\mu=30^{\circ}$, the free energy increases from $E_{\text {free }} / E_{\text {pot }}=\sin \left(30^{\circ}\right)^{2} \approx 0.25$ to a value of $E_{\text {free }} / E_{\text {pot }}=\sin \left(60^{\circ}\right)^{2} \approx 0.75$, or the total nonpotential energy changes from $E_{N} / E_{P}=1.25$ to $E_{N} / E_{P}=1.75$, which represents an increase of $40 \%$. This example may explain part of the discrepancy in free energies, which are about a factor of 4 higher when determined with a photospheric extrapolation code (such as the Wiegelmann NLFFF code used in Sun et 
al. 2012a), compared with the COR-NLFFF code that performs forward-fitting to coronal loops. The most plausible solution for the observed discrepancy in misalignment angles is that the photospheric magnetic field has about a factor of $\sqrt{4}=2$ higher azimuthally twisted field components in the non-forcefree zones than the coronal loops in the forcefree corona, which would produce a factor of 4 higher free energy for extrapolation codes that use the photospheric transverse field (which mostly contains the azimuthal twisted field component). Consequently, the coronal loops do not have a uniform twist all the way down to the photosphere, but increase their twist angle by a factor of about 2 at the photospheric footpoints.

\subsubsection{Non-forcefree Flare Dynamics}

Another possible reason for the apparent underestimate of the free energy during the X2.2 flare using the Wiegelmann code has been attributed to the dynamics of the eruption, which could possibly produce a strong deviation from a force-free state during the postflare phase (Sun et al. 2012a). However, the non-forcefreeness during the eruption should affect both the Wiegelmann NLFFF code and the COR-NLFFF forward-fitting method in a similar way, because both codes calculate the NLFFF solution under the assumption of forcefreeness, and thus it cannot explain the discrepancy in the free energy between the two codes. Moreover, the free energy calculated from photospheric data is up to a factor of 4 larger than the values calculated from forward-fitting of coronal data (Fig. 8d), measured at 1200 time steps during the entire time period of 5 days, and thus the discrepancy in free energies is persistently present, not just only during times of flaring or CMEs. However, inspecting Fig. 12b, there is a saturation apparent when the photospheric nonpotential energy reaches the highest values of $E_{N} \approx 1 \times 10^{33} \mathrm{erg}$, while the coronal free energies can exceed this limit, which may indicate a photospheric line-tying effect.

\subsubsection{Temporal Variability and Helicity Flux}

A striking difference is the time evolution of the free energy, as calculated at 1200 time steps over 5 days, is the degree of flucutations in the time profile $E_{\text {free }}(t)$ between the two codes (Fig. 8c, 8d). The time profile of the coronal COR-NLFFF code shows about an order of magnitude larger modulation depth than the photospheric Wiegelmann NLFFF code. Are those fluctuations real? The largest peaks and the decreases of the free energy $E_{\text {free }}(t)$ do indeed correlate with flaring events, such as evidenced by the GOES light curves (Figs. 8a) and the expanded time profiles of $E_{\text {free }}(t)$ (Fig. 10).

We compare the evolution of the nonpotentiality and helicity in the active region 11158 (from Fig. 12 and 14 in Liu and Schuck 2012). We show a juxtaposition of the time profiles of the free energy (obtained with the coronal COR-NLFFF code) and the helicity flux (Liu and Schuck 2012) in Fig. 14, which agree in the general trend of a systmatic increase over the 5 days of observations, while detailed correlations cannot be established due to the noise in the helicity flux (estimated to be $23 \%$ by Monte-Carlo simulations with the DAVE4VM code of Schuck 2008). In contrast, the photospheric (Wiegelmann) NLFFF code (Fig. 14a) shows a peak of the free energy at the beginning of the third day (during the largest flare), and then decreases during the next two days, which is different from the evolution of the coronal free energy (Fig. 14b) and the helicity flux (Fig. 14c). 


\section{CONCLUSIONS}

In this study we improved the accuracy and performance of the Coronal Non-Linear Force-Free Field (COR-NLFFF) forward-fitting code substantially by (1) implementing an automated loop tracing code for detection of coronal loops in multi-wavelength EUV images, which makes the manual or visual loop tracing unnecessary (Aschwanden 2010), and (2) by optimization of the forward-fitting technique to 2D loop coordinates, which relinquishes 3D reconstruction with stereoscopy (Aschwanden 2013c). In this study we applied the COR-NLFFF code to magnetic field modeling of AR 11158 during the time interval of 2011 Feb 12 to 17, which includes an X2.2 flare plus $35 \mathrm{M}$ and C-class flares. We calcuated the free magnetic energy over the 5 days with a cadence of 6 minutes and compare the results with standard NLFFF calculations using the Wiegelmann code with a cadence of 12 minutes. We compare quantitatively the magnitude and evolution of the free energy during the GOES-detected flares, in particular the detected decreases of free energy before and after the flares with both types of codes. The standard NLFFF codes (Wiegelmann 2004; Jiang and Feng 2013) use extrapolation of photospheric vector magnetograph data (with or without pre-processing), while our forward-fitting COR-NLFFF code uses coronal loop constraints. We compare also calculations with the virial theorem. The results are the following:

1. The total nonpotential magnetic energy measured during the X2.2 flare on 2011 Febr 15, 02 UT, agrees well $(\approx 15 \%)$ between photospheric standard NLFFF codes and the coronal forward-fitting code, i.e., $E_{N}=(10.4 \pm 1.4) \times 10^{32} \mathrm{erg}$. However, the total energy determinied with the virial theorem is about a factor of two lower, i.e., $E_{N}=(4.97 \pm 1.58) \times 10^{32} \mathrm{erg}$, probably due to a different computation box.

2. The potential energy measured during the X2.2 flare agrees also well $(\approx 10 \%)$ between the two types of codes, i.e., $E_{P}=(8.7 \pm 0.8) \times 10^{32}$ erg. The small difference, which is commensurable with the calibration of HMI and uncertainties among different NLFFF codes, corroborates the accuracy of the potential field modeling approach in terms of a a limited number of decomposed magnetic souces. The potential energy calculated with the virial theorem, $E_{P}=(2.43 \pm 0.78) \times 10^{32} \mathrm{erg}$, is a factor of 3 smaller, similar to the nonpotential energy, probably due to a different computation box.

3. The free magnetic energy measured during the X2.2 flare, i.e., the difference between the nonpotential and potential energy, disagrees by a factor of about two between the two types of codes. For standard NLFFF codes we find a value of in the range of $E_{\text {free }}=(1.65-2.4) \times 10^{32} \mathrm{erg}$, varying by about $15 \%$ with or without pre-processing, while we find a similar value of $E_{\text {free }}=1.0 \times 10^{32} \mathrm{erg}$ with the coronal forward-fitting COR-NLFFF code, so there is almost agreement between all codes in this case.

4. The most critical quantity is the decrease of free magnetic energy before and after the X2.2 flare, which sets an upper limit on the energy budget of flares. We find a decrease in the free energy by $\Delta E=E_{\text {post }}-E_{\text {pre }}=(0.37 \pm 0.04) \times 10^{32}$ erg with the standard photospheric NLFFF code (Sun et al. 2012a), which is about half of the decrease measured with the coronal forward-fitting CORNLFFF code, i.e., $\Delta E=E_{\text {post }}-E_{\text {pre }}=(0.62 \pm 0.28) \times 10^{32} \mathrm{erg}$, which is also consistent with the energy drop calculated with the virial theorem i.e., $\Delta E=E_{\text {post }}-E_{\text {pre }}=(1.05 \pm 1.04) \times 10^{32} \mathrm{erg}$ (Vemareddy et al. 2012), or $\Delta E=E_{\text {post }}-E_{\text {pre }}=0.84 \times 10^{32} \mathrm{erg}$ (Tziotziou et al. 2013). The fact that photospheric NLFFF codes measure a higher amount of free energy than the coronal NLFFF codes, but a too low decrease during flares, could partly be due to a smoothing and time-averaging effect of the pre-preprocessing algorithm.

5. The time evolution of the free energy, as calculated with a time resolution of 12 minutes over 5 days with both types, exhibits a systematic discrepancy in the amount of free energy obtained with the 
photospheric NLFFF versus the coronal NLFFF code. Since the free energy is dominated by the nonpotential transverse photospheric field, which corresponds to the azimuthal field component $B_{\varphi}$ in helically twisted field lines, it is conceivable that the twist of field lines is not uniform along their length, but could be stronger twisted by about a factor of $\sqrt{2}$ in the non-forcefree photosphere, and this way could explain why standard NLFFF codes produce a factor of $\approx 2$ higher free energies than coronal-fitting NLFFF codes.

6. Among the 36 GOES C,M, and X-class flares, we find a significant decrease in the free magnetic energy during the flares in 29 cases with the COR-NLFFF code. Similarly we find a significant decrease in many cases with the photospheric NLFFF code, however the energy drop is about a factor of $\approx 10$ smaller for the photospheric NLFFF code than for the coronal (COR-NLFFF) code, and thus confirms statistically the same trend as we found for the X2.2 flare.

7. The time evolution of the free energy in time steps of 12 minutes over 5 days exhibits about an order of magnitude stronger modulation depth for the coronal NLFFF code than for the photospheric NLFFF code, which is likely to be produced by the same effect that produces smaller energy decreases during the 36 flares, which could be partially be attributed to the smoothing effects of the pre-processing algorithm.

This study represents the first comparative test case between photospheric and coronal NLFFF codes, which revealed discrepancies resulting from the inconsistency between the photospheric non-forcefree and the coronal force-free field. It appears that the so far existing preprocessing algorthm that supposedly optimizes the photospheric field into a force-free field, is not consistent with the coronal force-field extrapolated downward to a photospheric altitude level. Therefore we conclude that the coronal field cannot be retrieved from photospheric information alone, but requires explicit information from the magnetic field in the force-free corona. While the present COR-NLFFF code used here is based on an analytical approximation that is accurate two second order, more advanced NLFFF codes that include the geometry of coronal loops should be developed in future. One code in this direction, using a quasi-Grad-Rubin scheme is under current development (Anna Malanushenko, private communication), using visually traced loops as input. Of course, ultimate objectivity can only be achieved with automated loop recognition algorithms.

\section{Appendix A: Parametric Study of the Numeric COR-NLFFF Code}

The default control parameters of the Coronal Non-linear Force-Free Field (COR-NLFFF) forwardfitting code used here are listed in Table 1. The accuracy of the forward-fitting results is primarily quantified with the median 2D misalignmen angle $\mu_{2}$ between the theoretical and observed (loop-aligned) magnetic field, obtained from the best forward-fit (which should converge to small values in the optimum case). Another test quantity is the ratio of the nonpotential to the potential energy, $q_{E}=E_{N} / E_{P}$, which should be a slowly-varying function of time during non-flaring episodes, and is expected to display a decrease during flares, the time interval when magnetic energy is dissipated. Therefore, in the following parametric study we demonstrate how these two quantities $\mu_{2}$ and $q_{E}$ vary as a function of the numerical input parameterers (Figs. 15 and 16).

All tests of this parametric study are performed with AIA and HMI data from 2011-February-15 in the time interval starting at 00:00:00 UT and ending $12 \mathrm{~s}$ later, in a field-of-view of $x=[-0.0268,0.3268]$ solar radii in East-West direction and $y=[-0.3885,-0.0885]$ in South-North direction. Since the COR-NLFFF 
code takes the full sphericity of the Sun into account, no transformation to Sun center is needed, as it would be required for most traditional NLFFF codes with a planar boundary of the computation box centered at Sun center.

Spatial resolution of magnetogram $\Delta x_{\text {mag }}$ (Figs. 15ab, 16ab): What is the ideal HMI resolution for our code? The HMI magnetogram has a full resolution of $\Delta x=0.00052$ solar radii. We rebin the magnetogram by factors of 1 to $10 \mathrm{HMI}$ pixels and show the obtained non-potential energy $E_{N}$ and potential energy $E_{P}$ as a function of the rebinned spatial resolution $\Delta x_{m a g}$ (Fig. 15a) and their ratio $q_{E}=E_{N} / E_{P}$ (Fig. 15b), respectively. We see a sharply peaked function $E_{N}\left(\Delta x_{m a g}\right.$ ) that peaks at $\Delta x_{m a g}=0.0015$ (or 3 HMI pixels). We can understand this function as a combination of two effects, i.e., (i) the undersampling of overresolved structures in the magnetogram in the case of $\lesssim 3$ HMI pixels, and (ii) over-smoothing of unresolved structures in the case of $\gtrsim 3 \mathrm{HMI}$ pixels. The undersampling results because of the limited number of modeled magnetic source structures (typically $n_{m a g} \approx 100$ ) in magnetograms, which cover a full-resolution size of $577 \times 577=332,929$ pixels, with a sizable fraction $n_{\text {pixel }}$ containing a significant magnetic field strength. The limitation of $n_{\text {mag }} \ll n_{\text {pixel }}$ of the code causes an underestimate of both the potential as well as the nonpotential magnetic energy at high spatial resolution. If we approximate the area with high magnetic field strengths with a mean value $B$, the total magnetic energy is expected to scale as $E_{m a g}=n_{m a g} B^{2} \Delta x_{m a g}^{2}$ for $n_{m a g} \ll n_{\text {pixel }}$, which is a quadratic function of the pixel size and fits the observed function in the range of $\Delta x_{m a g}=1, \ldots, 3$ pixels (left red curve in Fig. 15a). On the other side, above the critical limit where the number of model components is sufficient to cover the number of macropixels, i.e., $n_{\text {mag }}>n_{\text {pixel }}$, we expect that the rebinning conserves the magnetic flux $\Phi=\sum B \Delta x_{\text {mag }}^{2}$, but the rebinned field strength $B_{\text {rebin }}$ scales then reciprocally with the macropixel size, i.e., $B_{\text {rebin }}=B / \Delta x_{m a g}$, which leads to a quadratic decrease of the magnetic energy as a function of the (rebinned) macro pixel size, i.e., $E_{\text {mag }} \propto\left(\Delta x_{\text {mag }}\right)^{-2}$ (right red curve in the range of 3-10 HMI pixels in Fig. 15a). Therefore, the least biased magnetic energy is measured at the critical limit $n_{\text {mag }} \approx n_{\text {pixel }}$, which turns out to be 3 HMI pixels here (or $\left.\approx 1.5^{\prime \prime} \approx 1500 \mathrm{~km}\right)$. In other words, the critical scale of $\Delta x_{m a g} \approx 1500 \mathrm{~km}$ represents the optimum scale where the number of model sources match the number of significant magnetic sources, so that the side effects of under-resolving and over-resolving dissappear. Hence we are using rebinned magnetograms with $\Delta x_{m a g}=3$ HMI pixels throughout this study.

The coverage of the fitted magnetic areas can be seen from the circles displayed in the bottom panels of Fig. 17, for three different resolution scales (1, 3, and 10 HMI pixels). The spatial resolutions shown in Fig. 17 are $\left(\Delta x_{m a g}=0.0005,0.0015\right.$, and 0.005 solar radii). We find a maximum in the detection of nonpotential magnetic energy of $E_{N}=8.6 \times 10^{32} \mathrm{erg}$, a potential energy of $E_{P}=7.6 \times 10^{32} \mathrm{erg}$, a free energy of $E_{\text {free }}=E_{N}-E_{P}=1.0 \times 10^{32} \mathrm{erg}$, which corresponds to an energy ratio of $q_{E}=1.13$ (Fig. 15b), or a free energy of $13 \%$ of the potential energy. These values agree with other studies using the traditional NLFFF method, i.e., $E_{N} \approx 10 \times 10^{32} \mathrm{erg}$ (Sun et al. 2012a; Jiang and Feng 2013). The mean misalignment angle of the best-fit non-potential field using our COR-NLFFF model is $\mu \approx 5.0^{\circ}$ (Fig. 16b), while the potential field has a misalignment of $\mu \approx 10.0^{\circ}$ (Fig. 16a).

Number of magnetic sources $n_{\operatorname{mag}}$ (Fig. 15c, 16c): What is the optimim number of magnetic sources that is needed for the decomposition of the LOS magnetogram and construction of the model map? In the three examples shown in Fig. 17 we used $n_{m a g}=100$ magnetic sources, which appears to be sufficient to represent most of the magnetic flux down to a level of a few percent, even for this case of a complex active region two hours before an X-class flare. We varied the number of decomposed magnetic sources from $n_{\text {mag }}=10$ to $n_{\text {mag }}=200$ and found a quite robust behavior in the best-fit value of the nonpotential energy ratio $q_{E} \approx 1.13$ for $n_{m a g} \gtrsim 50$ (Fig. $15 \mathrm{c}$ ), with a mean misalignment angle of $\mu \approx 5.0^{\circ}$ (Fig. 16c). We choose 
$n_{\text {mag }}=100$ as default value.

Threshold level in loop detection $q_{\text {med }}$ (Fig. 15d, 16d): The threshold level of automated loop detection in different wavelength filters is empirically determined by visual inspection of false loop detections in noisy image areas. We find a good discrimination for the following factors of the median flux (obtained from the boundary areas of the highpass-filtered EUV images): $q_{m e d, 0}(\lambda)=[5,2,0,0,1,5,5]$ for the wavelengths $\lambda=[94,131,171,193,211,304,335] \AA$. In our parametric study we vary these median levels by a factor of $q_{m e d}=0.2, \ldots, 2.0 \times q_{m e d, 0}$. The obtained energy ratios $q_{\text {free }}$ (Fig. 15d) and misalignment angles $\mu_{2}$ (Fig. 16d) show almost invariant values as a function of the flux threshold variation, which corroborates the robustness of our loop detection scheme.

Loop selection by wavelength $\lambda$ (Fig. 15e, 16e): We vary now the parameters that affect the selection of loops (Section 3.2). Let us first consider the different wavelength filters $(\lambda)$. The nonpotential energy ratios $q_{E}=E_{N} / E_{P}$ retrieved from each single wavelength separatetly are shown in Fig. 15e, which obviously demonstrates that not each wavelength contains a representative subset of nonpotential loops. If we combine the loop tracings from all 7 wavelength filters, we find a value of $q_{E}=1.13$ (or $13 \%$ free energy), which is approximately retrieved by those wavelength filters that contain representative subsets of the loops (Fig. 15e). However, some filters yield values that deviate substantially from the mean, and thus seem not to contain a representative sample of loops. Investigating the misalignment angles $\mu_{2}$ for these seven filters (Fig. 16e), we see that the best-fit values in each wavelength converge to a range of $\mu_{2} \approx 4^{\circ}-7^{\circ}$, while the synthesized set of loops from all wavelengths yields $\mu_{2} \approx 5^{\circ}$. We conclude that a well-balanced sample of loops synthesized from all wavelength filters is required to obtain the correct free energy.

Maximum number of loops per filter $n_{\text {loop }}$ (Figs. 15f, 16f): If we combine all seven filters, we obtain about 1000 loop structures. If we limit the maximum number of loops extracted per wavelength filter to $n_{\text {loop }}=100$, we obtain 452 loops, which reduces the computation time by a factor of two. The parametric study shown in Fig. $15 \mathrm{f}$ shows that we can reduce the number down to $n_{\text {loop }} \approx 40$ without changing the result of the nonpotential energy. Only below that limit we loose too much potential loops (mostly in the 171 and $193 \AA$ images) so that the sample is not representative anymore. Thus we choose $n_{\text {loop }}=50$ as default value. The misalignment angle is nearly insensitive to the maximum number of selected loops per wavelength (Fig. 16f).

Minimum length of selected loops $l_{\min }$ (Figs. $15 \mathrm{~g}, 16 \mathrm{~g}$ ): Besides the minimum loop length criterion defined in the loop tracing algorithm, we can additionally set a minimum loop length criterion $l_{\text {min }}$ for the subset of loops selected for forward-fitting. This allows us to balance the relative ratio of short loops (which occur in the core of active regions and exhibit generally a higher nonpotential energy) and long loops (which occur in the outer shells of active regions and exhibit a more potential field characteristics). The parametric study in the range of $l_{\min }=[10,100]$ pixels exhibits for $l_{\min } \lesssim 20$ pixels an overabundance of short loops, producing a relatively high nonpotential energy ratio $q_{E} \approx 1.2$, and inversely, an overabundance of long loops for $l_{\text {min }} \gtrsim 40$ pixels, which are mostly potential-like and produce a too low nonpotential energy ratio $q_{E} \lesssim 1.1$ (Fig. 15g). Thus, we choose an intermediate value of $l_{\min }=30$ as default. The misalignment angle varies in the range of $\mu_{2} \approx 3^{\circ}-6^{\circ}$ in this parameter range (Fig. 16g).

Loop curvature radius $r_{\min }$ (Figs. 15h, 16h): The automated loop tracing code picks curvi-linear structures with a minimum curvature radius $r_{\min }$, while structures with shorter curvature radii often result from "curved chains" of moss structure (Berger et al. 1999). Tests with curvature radii varied from $r_{\text {min }}=10$ to 60 pixels reveal no systematic tendency of the best-fit non-potential energy ratio $q_{E}=E_{N} / E_{P}$ (Fig. 16h) or misalignment angle. Thus we choose $r_{\min }=30$ pixels as default value. 
Suppression of false loop structures: The most common false loop structures are: (1) curved chains of moss features, (2) saturated pixels during strong flares, (3) vertical streaks from pixel bleeding in the CCD camera during strong flares, and (4) diffraction patterns from the entrance mesh filter during intense flares. Features (1) and (4) can mostly be filtered out by a minimum loop length requirement (which is choosen here to be $l_{\text {min }} \geq 30$ AIA pixels). Loop structures containing saturated pixels are rejected based on their flux value $\left(F>2^{14}-1 \mathrm{DN} / \mathrm{s}\right)$. Vertical streaks from pixel bleeding are also easily detected by their small variation in x-coordinates (less than one pixel, either in the northern or southern half of the automatically detected structures). The rejection of false loop structures improves the best-fit solution significantly, as we tested in images during flare peaks.

The number of loop segment points $n_{\text {seg }}$ (Fig. 15i, 16i): Forward-fitting is carried out at $n_{f i t}=n_{L} \times$ $n_{\text {seg }}$ loop $2 \mathrm{D}$ positions $\left(x_{i}, y_{i}\right), i=1, \ldots, n_{f i t}$, where $n_{f}$ is the number of selected loops, and $n_{\text {seg }}$ is the number of loop segments per loop, distributed equidistantly along the automatically traced loop segments. A too small number of loop segments may not be sufficient for a representative fit, and a too large number consumes more computation time. The parametric study in Fig. 15i and 16i shows that the nonpotential energy is robustly retrieved for $n_{\text {seg }}=3$ to 15 . We choose $n_{\text {seg }}=7$ as default value.

The force-free $\alpha$-parameter increment $\Delta \alpha$ (Fig. 15j): The $\alpha$-parameter is varied for each magnetic source within a range of $\pm \Delta \alpha$ for every magnetic source in each iteration cycle. If we choose $\Delta \alpha$ too small, we need a too large number of iterations to converge, requiring too much computation time. If we choose it too large, the minimization of the misalignment angle per iteration misses the local maximum and will fluctuate erratically instead of converging steadily to the absolute minimum. The parametric study in Fig. $15 \mathrm{j}$ and $15 \mathrm{j}$ show that the nonpotential energy is stably retrieved in the range of $\Delta \alpha \approx 5-100\left[R_{\odot}^{-1}\right]$. We choose $\Delta \alpha=10\left[R_{\odot}^{-1}\right]$ as default value.

The altitude range of the computation box $h_{\max }$ (Figs. $15 \mathrm{k}$ and $15 \mathrm{k}$ ): The height of the computation box can possibly affect the forward-fitted loop solutions, because larger heights contain more ambiguities in the reconstruction of the line-of-sight coordinate $z_{i}$ for each of the fitting points. In addition, increasing the altitude yields a slightly higher amount of volume-integrated magnetic energy. The parametric study shown in Figs. 15k and 15k, however, shows an invariant retrieval of the nonpotential energy for altitude ranges of $h_{\max }=0.05-0.25$ solar radii. We choose $h_{\max }=0.15$ as default value.

The maximum number of iterations $n_{\text {iter }}$ (Figs. 15h, 16h): Our chosen forward-fitting method is the Powell method, which determines the local minimum for each free parameter $\alpha_{j}$ sequentially per iteration cycle. An example of the change of misalignment angle $\mu$ and nontpotential energy ratio $q_{E}=E_{N} / E_{P}$ as a function of the iteration cycle is shown in Fig. $15 \mathrm{l}$ and $15 \mathrm{l}$, for $n_{\text {iter }}=1-10$. The misalignment angle starts with $\mu \approx 9^{\circ}$ for the potential-field solution in the first iteration, and converges to a final best-fit value of $\mu \approx 5^{\circ}$ after the fourth iteration. Thus we choose $n_{\text {iter }}=6$ as default value, since more iterations were found not to improve the goodness-of-fit significantly.

The author appreciates the constructive comments by an anonymous referee and helpful discussions with Allen Gary, Anna Malanushenko, Marc DeRosa, and Karel Schrijver. Part of the work was supported by NASA contract NNG 04EA00C of the SDO/AIA instrument and the NASA STEREO mission under NRL contract N00173-02-C-2035. 


\section{REFERENCES}

Altschuler, M.D. and Newkirk, G.Jr. 1969, Solar Phys. 9, 131.

Alvarado-Gomez, J.D., Buitrago-Casas, J.C., Martinez-Oliveros, J.C., Lindsey, C., Hudson, H., and CalvoMozo, B. 2012, Solar Phys. 280, 335.

Aschwanden, M.J., Lee,J.K., Gary,G.A., Smith,M., and Inhester,B. 2008, Solar Phys. 248, 359.

Aschwanden, M.J. 2010, Solar Phys. 262, 399.

Aschwanden, M.J. and Sandman, A.W. 2010, AJ 140, 723.

Aschwanden, M.J., Wülser, J.P., Nitta, N.V., Schrijver, C.J., DeRosa, M., and Malanushenko, A. 2012, ApJ $756,124$.

Aschwanden, M.J. and Freeland, S.L. 2012, ApJ, 754, 112.

Aschwanden, M.J. 2013a, Sol. Phys., 287, 323.

Aschwanden, M.J. and Malanushenko, A. 2013, Sol. Phys., 287, 345.

Aschwanden, M.J. 2013b, Sol. Phys., 287, 369.

Aschwanden, M.J. 2013c, ApJ 763, 115.

Aschwanden, M.J., Boerner, P., Schrijver, C.J., Malanushenko, A. 2013a, Sol. Phys.283, 5.

Aschwanden, M.J., De Pontieu, B., and Katrukha, A. 2013b, Entropy 15(8), 3007.

Beauregard, L., Berma, M., and Denker, C. 2012, Astron.Nachr. 333/2. 125.

Berger, T.E., De Pontieu, B., Fletcher, L., Schrijver, C.J., tarbell, T.D., and Title, A.N. 1999, Solar Phys. 190, 409.

Bobra, M.G., van Ballegooijen, A.A., and DeLuca, E.E. 2008, ApJ 672, 1209.

Boerner, P., Edwards, C., Lemen, J., Rausch, A., Schrijver, C., Shine, R., Shing, L., Stern, R., Tarbell, T. et al. 2012, Sol. Phys., 275, 17.

Borovik, V.N., Grigoreva, I.Y., and Korzhavin, A.N. 2012, Geomagn. Aeronomy 52/8, 1032.

Chandrasekhar, S. (ed.) 1961, Hydrodynamic and Hydromagnetic Stability, (Oxford: Clerendon).

Chen, N., Ip, W.H., Innes, D. 2013, ApJ 769, 96.

DeRosa, M.L., Schrijver, C.J., Barnes, G., Leka, K.D., Lites, B.W., Aschwanden, M.J., Amari, T., Canou, A., et al. 2009, ApJ 696, 1780.

Emslie, A.G., Kucharek, H., Dennis, B.R., Gopalswamy, N., Holman, G.D., Share, G.H., Vourlidas, A., Forbes, T.G., et al. 2004, JGR 109, A10104.

Emslie, A.G., Dennis, B.R., Holman, G.D., and Hudson, H.S. 2005, JGR 110, A11103.

Emslie, A.G., Dennis, B.R., Shin, A.Y., Chamberlin, P.C., Mewaldt, R.A., Moore, C.S., Share, G.H., Vourlidas, A., and Welsch, B.T. 2012, ApJ 759, 71.

Feng, L., Wiegelmann, T., Su, Y., Inhester, B., Li, Y.P., Sun X.D., and Gan, W.Q. 2013, ApJ 765, 37.

Gary, G.A., Hu, Q., and Lee, J.K. 2014a, Sol. Phys. 289, 847.

Gary, G.A., Hu, Q., Lee, J.K., and Aschwanden, M.J. 2014b, Sol. Phys. (in press).

Gosain. S. 2012, ApJ 749, 85.

Guo, Y., Ding, M.D., Wiegelmann, T., Li, H. 2008, ApJ 679, 1629. 
Guo, Y., DIng, M.D., Schmieder, B., Li, H., Török, T., and Wiegelmann, T. 2010, ApJ 725, 38.

Hoeksema, J.T., Liu, Y., Hayashi, K., Sun, X., Schou, J., Couvidat, S., Norton, A., Bobra, M., et al. 2014, Solar Phys (subm.).

Inoue, S., Kusano, K., Magara, T., Shiota, D., Yamamoto, T.T. 2011, ApJ 738, 161.

Inoue, S., Magara, T., Watari, S., Choe, G.S. 2012, ApJ 747, 126.

Inoue, S., Hayashi, K., Shiota, D., Magara, T., and Choe, G.S. 2013, ApJ 770, 79.

Jiang, C. and Feng, X. 2013, ApJ 769, 144.

Jiang, Y., Zheng, R., Yang, J., Hong, J., Yi, B., Yang, D. 2012, ApJ 744, 50.

Jing, J., Park, S.H., Liu, C., Lee, J.W., Wiegelmann, T., Xu, Y., Deng, N., and Wang, H. 2012, ApJ 752, L9.

Kosovichev, A.G. 2011, ApJ 734, L15.

Kusano, K., Bamba, Y., Yamamoto, T.T., Iida, Y., Toriumi, S., and Asai, A. 2012, ApJ 760, 31.

Lemen, J.R., Title, A.M., Skin, D.J., Boerner, P.F., Chou, C., Drake, J.F., Duncan, D.W., Edwards, C.G. et al. 2012, Sol. Phys., 275, 17.

Li, H., Schmieder, B., Song, M.T., Bommier, V. 2007, AA 475, 108.

Liu, C., Deng, N., Liu,R., Lee, J.W., Wiegelmann, T., Jing, J., Xu, Y. 2012, ApJ 745, 4.

Liu, Y. and Schuck, P.W. 2012, ApJ 761, 105.

Liu, Y., Zhao, J.W., Schuck, P.W. 2013, Solar Phys. 287, 279.

Malanushenko, A., Yusuf, M.H., and Longcope, D.W. 2011, ApJ 736, 97.

Malanushenko, A., Schrijver, C.J., DeRosa, M.L., Wheatland, M.S., and Gilchrist, S.A. 2012, ApJ 756, 153.

Maricic, D., Bostasyan, N., Dumbovic, M., Chilingarian, A., Mailyan, B., Rostomyan, H., Arakelyan, K., Vrsnak, B., et al. 2012, J.Phys.Conf.Ser. 409, 2158.

Maricic, D., Vrsnak, B., Dumbovic, M., Zic, T., Rosa, D., Hrzina, D., Lulic, S. Romstajn, I., et al. 2013, Solar Phys. (in press).

Maurya, R.A., Vemareddy, P,, and Ambastha, A. 2012, ApJ 747, 134.

Metcalf, T.R., DeRosa, M.L., Schrijver, C.J., Barnes, G., Van Ballegooijen, A.A., Wiegelmann, T., Wheatland, M.S., Valori, G., and McTiernan, J.M. 2008, Sol. Phys., 247, 269.

Milligan, R.O., Chamberlin, P.C., Hudson, H.S., Woods, T.N., Mathioudakis, M., Fletcher, L., Kowalski, A.F., and Keenan, F.P. 2012, ApJ 748, 14.

Olmedo, O., Vourlidas, A., Zhang, J., and Cheng, X. 2012, ApJ 756, 143.

Petrie, G.J.D. 2012a, Solar Phys. 287, 415.

Petrie, G.J.D. 2012b, ApJ 759, 50.

Pesnell, W.D., Thompson, B.J., and Chamberlin, P.C. 2012, Solar Phys. 275, 3.

Regnier, S. 2013, Solar Phys. online-first, DOI:10.1007/s11207-013-0367-8.

Sandman, A.W. and Aschwanden, M.J. 2011, Sol. Phys., 270, 503.

Savcheva, A., Pariat, E., van Ballegooijen, A.A., Aulanier, G., and DeLuca E.E. 2012a, ApJ 750, 15.

Savcheva, A., van Ballegooijen, A.A, DeLuca, E.E. 2012b, ApJ 744, 78. 
Savcheva, A.S., Green, L.M., Van Ballegooijen, A.A., DeLuca, E.E. 2012c, ApJ 759, 105.

Scherrer, P.H., Schou, J., Bush, R.I., Kosovichev, A.G., Bogart, R.S., Hoeksema, J.T., Liu, Y., Duvall, T.L., Zhao, J., Title, A.M., Schrijver, C.J., Tarbell, T.D., and Tomczyk, S. 2012, Solar Phys. 275, 207.

Schrijver, C.J., DeRosa, M.L., Metcalf, T., Barnes, G., Lites, B., Tarbell, T., McTiernan, J., Valori, G., et al. 2008, ApJ 675, 1637.

Schrijver, C.J., Aulanier, G., Title, A.M., Pariat, E., Delannée, C. 2011, ApJ 738, 167.

Schuck, P.W. 2008, ApJ 683, 1134.

Shen, J., Ji, H., Wiegelmann, T., and Inhester, B. 2013, ApJ 764, 86.

Song, Q., Zhang, J., Yang, S.H., and Liu,Y. 2013, Res.Astron.Astrophys. 13/2, 226.

Su, Y., Van Ballegooijen, A.A., Lites, B.W., DeLuca, E.E., Golub, L., Grigis, P.C., Guangli, H., Ji, H.S. 2009a, ApJ 691, 105.

Su, Y., Van Ballegooijen, A.A., Schmieder, R., Berlicki, A., Guo,Y., Golub, L., Guangli, H., 2009b, ApJ 704, 341.

Su, Y., Surges, V., van Ballegooijen, A., DeLuca, E., and Golub, L. 2011, ApJ 734, 53.

Su, J.T., Liu, Y., Shen, Y.D., Liu, S., and Mao, X.J. 2012, ApJ 760, 82.

Sun, X., Hoeksema, J.T., Liu, Y., Wiegelmann, T., Hayashi, K., Chen, Q., and Thalmann, J. 2012a, ApJ $748,77$.

Sun, X., Hoeksema, J.T., Liu, Y., Chen, Q., and Hayashi, K. 2012b, ApJ 757, 149.

Tarr, L., Longcope, D., and Millhouse, M. 2013, ApJ 770, 4.

Toriumi, S., Iida, Y., Bamba, Y., Kusano, K., Imada, S., and Inoue, S. 2013, ApJ 773, 128.

Vemareddy, P., Ambastha, A., Maurya, R.A., and Chae, J. 2012a, ApJ 761, 86.

Vemareddy, P., Ambastha, A., and Maurya, R.A. 2012b, ApJ 761, 60.

Wang, S., Chang, L., Liu, R., Deng, N., Yang, L., Wang, H. 2012, ApJ 745, L17.

Wang, S., Liu, C., and Wang, H. 2013, ApJ (subm.).

Wang, R., Yan, Y., and Tan, B. 2014, Solar Phys. (subm).

Welsch, B.T., Fisher, G.H., and Sun, X. 2013, ApJ 765, 98.

Wiegelmann, T. 2004, Solar Phys. 219, 87.

Wiegelmann, T., Inhester, B., and Sakurai, T. 2006, Solar Phys. 233, 215.

Wiegelmann, T., Thalmann, J.K., Schrijver, C.J., DeRosa, M.L., and Metcalf, T.R. 2008, Solar Phys. 247, 249 .

Wheatland, M.S. and Regnier, S. 2009, ApJ 700, L88.

Yamamoto, T.T. and Kusano, K. 2012, ApJ 752, 126.

Yashiro, S., Gopalswamy, N., Mäkelä, P., Akiyama, S., Uddin, W., Srivastava, A.K., Joshi, N.C., Chandra, R., et al. 2014, Adv.Res.Spac.Sci. (in press).

Young, P.R., Doschek, G.A., Warren, H.P., and Hara, H. 2013, ApJ 766, 127.

Zharkov, S., Green, L.M., Matthews, S.A., and Zharkova, V.V. 2011, ApJ 741, 35.

Zharkov, S., Green, L.M., Matthews, S.A., and Zharkova, V.V. 2013, Solar Phys. 284, 315. 
Tziotziou, K., Georgoulis, M.K., and Liu, Y. 2013, ApJ 772, 115. 
Table 1. Default control parameters of the coronal non-linear force-free field (COR-NLFFF) forward-fitting code used in this study.

\begin{tabular}{cll}
\hline \hline \multicolumn{1}{c}{ Task: } & \multicolumn{1}{c}{ Control parameter } & \multicolumn{1}{c}{ Value } \\
\hline \multirow{2}{*}{ Data selection: } & Date and time of observation & 2011 Feb 15, 00:00:00 UT \\
& Instruments & AIA, HMI (SDO) \\
& East-west field-of-view & {$[0.0268,0.3268] R_{\odot}$} \\
& North-south field-of-view & {$[-0.3885,-0.0885] R_{\odot}$} \\
& AIA wavelengths & $94,131,171,193,211,304,335 \AA$ \\
& spatial pixel size & $\Delta x_{A I A}=0.6^{\prime \prime}$ \\
& Lowpass filter boxcar & $n_{\text {sm1 }}=5$ pixels \\
& Highpass filter boxcar & $n_{\text {sm } 2}=7$ pixels \\
& Image base level/median & $q_{\text {med }}=5,2,0,0,1,5,5$ \\
& minimum loop length & $l_{\text {min }}=30$ pixels \\
& minimum loop curvature radius & $r_{\text {min }}=30$ pixels \\
& field line step & $d_{\text {field }}=0.002 R_{\odot}$ \\
Magnetic sources: & rebinned pixel size & $\Delta x_{\text {mag }}=3$ pixel $\left(1.5^{\prime \prime}, 0.0015 R_{\odot}\right)$ \\
& number of magnetic sources & $n_{\text {mag }}=100$ \\
Loop selection: & maximum number per filter & $n_{\text {loop }}=50$ \\
Forward-Fitting: & minimization iteration method & Powell \\
& minimum number of iterations & $n_{\text {iter } \text { min }}=3$ \\
& maximum number of iterations & $n_{\text {iter }, \text { max }}=6$ \\
& maximum height & $h_{\text {max }}=0.15 R_{\odot}$ \\
& number of loop segments & $n_{\text {seg }}=7$ \\
& $\alpha$-parameter increment & $\Delta \alpha=10 R_{\odot}^{-1}$ \\
\hline
\end{tabular}


Table 2. Free magnetic energy and changes during X,M,C-class flares (\#1-36), calculated (a) with the COR-NLFFF code and (b) with the Wiegelmann NLFFF code (Sun et al. 2012a). The preflare free energies are $E_{\text {pre }}^{a}$ nad $E_{\text {pre }}^{b}$, the postflare values are $E_{\text {post }}^{a}$ and $E_{\text {post }}^{b}$, and the changes during the flare are $\Delta E^{a}$ and $\Delta E^{b}$, in units of $10^{30} \mathrm{erg}$.

\begin{tabular}{|c|c|c|c|c|c|c|c|c|}
\hline $\begin{array}{c}\text { Flare } \\
\quad \#\end{array}$ & $\begin{array}{c}\text { Observation } \\
\text { Date }\end{array}$ & $\begin{array}{l}\text { GOES } \\
\text { class }\end{array}$ & $\begin{array}{c}E_{p r e}^{a} \\
\left(10^{30} \text { erg }\right)\end{array}$ & $\begin{array}{c}E_{\text {post }}^{a} \\
\left(10^{30} \mathrm{erg}\right)\end{array}$ & $\begin{array}{c}\Delta E^{a} \\
\left(10^{30} \mathrm{erg}\right)\end{array}$ & $\begin{array}{c}E_{p r e}^{b} \\
\left(10^{30} \text { erg }\right)\end{array}$ & $\begin{array}{c}E_{\text {post }}^{b} \\
\left(10^{30} \mathrm{erg}\right)\end{array}$ & $\begin{array}{c}\Delta E^{b} \\
\left(10^{30} \mathrm{erg}\right)\end{array}$ \\
\hline 1 & 2011-Feb-13 13:43 & $\mathrm{C} 4.7$ & $26 \pm 6$ & $10 \pm 6$ & $-15 \pm 9$ & $129 \pm 4$ & $129 \pm 4$ & $0 \pm 2$ \\
\hline 2 & 2011-Feb-13 17:27 & M6.6 & $65 \pm 6$ & $21 \pm 6$ & $-44 \pm 9$ & $164 \pm 4$ & $142 \pm 4$ & $-21 \pm 2$ \\
\hline 3 & 2011-Feb-13 21:16 & $\mathrm{C} 1.1$ & $76 \pm 6$ & $25 \pm 6$ & $-51 \pm 9$ & $167 \pm 4$ & $157 \pm 4$ & $-9 \pm 1$ \\
\hline 4 & 2011-Feb-14 2:35 & C1.6 & $46 \pm 10$ & $38 \pm 10$ & $-7 \pm 14$ & $190 \pm 4$ & $190 \pm 4$ & $0 \pm 3$ \\
\hline 5 & 2011-Feb-14 4:29 & $\mathrm{C} 8.3$ & $52 \pm 10$ & $29 \pm 10$ & $-23 \pm 14$ & $196 \pm 4$ & $181 \pm 4$ & $-15 \pm 2$ \\
\hline 6 & 2011-Feb-14 6:50 & C6.6 & $75 \pm 10$ & $42 \pm 10$ & $-33 \pm 14$ & $201 \pm 4$ & $200 \pm 4$ & $0 \pm 2$ \\
\hline 7 & 2011-Feb-14 8:38 & $\mathrm{C} 1.8$ & $95 \pm 10$ & $40 \pm 10$ & $-54 \pm 14$ & $209 \pm 4$ & $198 \pm 4$ & $-11 \pm 2$ \\
\hline 8 & 2011-Feb-14 11:50 & $\mathrm{C} 1.7$ & $44 \pm 10$ & $30 \pm 10$ & $-14 \pm 14$ & $213 \pm 4$ & $210 \pm 4$ & $-3 \pm 1$ \\
\hline 9 & 2011-Feb-14 12:40 & C9.4 & $70 \pm 10$ & $33 \pm 10$ & $-37 \pm 14$ & $219 \pm 4$ & $206 \pm 4$ & $-13 \pm 1$ \\
\hline 10 & 2011-Feb-14 13:46 & $\mathrm{C} 7.0$ & $70 \pm 10$ & $46 \pm 10$ & $-24 \pm 14$ & $217 \pm 4$ & $208 \pm 4$ & $-8 \pm 1$ \\
\hline 11 & 2011-Feb-14 17:20 & M2.2 & $60 \pm 10$ & $34 \pm 10$ & $-25 \pm 14$ & $244 \pm 4$ & $228 \pm 4$ & $-16 \pm 3$ \\
\hline 12 & 2011-Feb-14 19:23 & C6.6 & $52 \pm 10$ & $37 \pm 10$ & $-14 \pm 14$ & $250 \pm 4$ & $241 \pm 4$ & $-9 \pm 3$ \\
\hline 13 & 2011-Feb-14 23:14 & $\mathrm{C} 1.2$ & $77 \pm 10$ & $44 \pm 10$ & $-32 \pm 14$ & $264 \pm 4$ & $259 \pm 4$ & $-5 \pm 2$ \\
\hline 14 & 2011-Feb-14 23:39 & $\mathrm{C} 2.7$ & $62 \pm 10$ & $40 \pm 10$ & $-21 \pm 14$ & $265 \pm 4$ & $259 \pm 4$ & $-5 \pm 2$ \\
\hline 15 & 2011-Feb-15 0:31 & $\mathrm{C} 2.7$ & $92 \pm 20$ & $61 \pm 20$ & $-31 \pm 28$ & $259 \pm 6$ & $250 \pm 6$ & $-8 \pm 4$ \\
\hline 16 & 2011-Feb-15 1:43 & $\mathrm{X} 2.2$ & $98 \pm 20$ & $36 \pm 20$ & $-62 \pm 28$ & $256 \pm 6$ & $218 \pm 6$ & $-37 \pm 4$ \\
\hline 17 & 2011-Feb-15 4:26 & $\mathrm{C} 4.8$ & $61 \pm 20$ & $56 \pm 20$ & $-5 \pm 28$ & $241 \pm 6$ & $230 \pm 6$ & $-11 \pm 2$ \\
\hline 18 & 2011-Feb-15 10: 1 & C1.0 & $150 \pm 20$ & $53 \pm 20$ & $-97 \pm 28$ & $214 \pm 6$ & $213 \pm 6$ & $0 \pm 3$ \\
\hline 19 & 2011-Feb-15 14:31 & $\mathrm{C} 4.8$ & $179 \pm 20$ & $39 \pm 20$ & $-139 \pm 28$ & $198 \pm 6$ & $193 \pm 6$ & $-4 \pm 1$ \\
\hline 20 & 2011-Feb-15 18: 6 & $\mathrm{C} 1.7$ & $147 \pm 20$ & $59 \pm 20$ & $-88 \pm 28$ & $222 \pm 6$ & $208 \pm 6$ & $-14 \pm 3$ \\
\hline 21 & 2011-Feb-15 19:30 & C6.6 & $122 \pm 20$ & $47 \pm 20$ & $-75 \pm 28$ & $223 \pm 6$ & $204 \pm 6$ & $-18 \pm 3$ \\
\hline 22 & 2011-Feb-15 22:48 & $\mathrm{C} 1.3$ & $87 \pm 20$ & $26 \pm 20$ & $-61 \pm 28$ & $222 \pm 6$ & $209 \pm 6$ & $-13 \pm 5$ \\
\hline 23 & 2011-Feb-16 0:57 & $\mathrm{C} 2.0$ & $103 \pm 34$ & $50 \pm 34$ & $-53 \pm 48$ & $214 \pm 6$ & $214 \pm 6$ & $0 \pm 3$ \\
\hline 24 & 2011-Feb-16 1:31 & M1.0 & $207 \pm 34$ & $81 \pm 34$ & $-126 \pm 48$ & $205 \pm 6$ & $204 \pm 6$ & $0 \pm 2$ \\
\hline 25 & 2011-Feb-16 1:55 & $\mathrm{C} 2.2$ & $136 \pm 34$ & $78 \pm 34$ & $-58 \pm 48$ & $213 \pm 6$ & $203 \pm 6$ & $-9 \pm 2$ \\
\hline 26 & 2011-Feb-16 5:39 & C5.9 & $118 \pm 34$ & $93 \pm 34$ & $-25 \pm 48$ & $219 \pm 6$ & $202 \pm 6$ & $-16 \pm 2$ \\
\hline 27 & 2011-Feb-16 6:18 & $\mathrm{C} 2.2$ & $131 \pm 34$ & $70 \pm 34$ & $-61 \pm 48$ & $225 \pm 6$ & $197 \pm 6$ & $-28 \pm 3$ \\
\hline 28 & 2011-Feb-16 7:34 & M1.1 & $182 \pm 34$ & $58 \pm 34$ & $-123 \pm 48$ & $205 \pm 6$ & $205 \pm 6$ & $0 \pm 4$ \\
\hline 29 & 2011-Feb-16 9: 1 & C9.9 & $131 \pm 34$ & $49 \pm 34$ & $-82 \pm 48$ & $224 \pm 6$ & $191 \pm 6$ & $-32 \pm 3$ \\
\hline 30 & 2011-Feb-16 10:25 & $\mathrm{C} 3.2$ & $61 \pm 34$ & $22 \pm 34$ & $-39 \pm 48$ & $202 \pm 6$ & $201 \pm 6$ & $0 \pm 3$ \\
\hline 31 & 2011-Feb-16 11:58 & C1.0 & $83 \pm 34$ & $16 \pm 34$ & $-66 \pm 48$ & $199 \pm 6$ & $191 \pm 6$ & $-7 \pm 3$ \\
\hline 32 & 2011-Feb-16 14:18 & M1.6 & $192 \pm 34$ & $34 \pm 34$ & $-157 \pm 48$ & $181 \pm 6$ & $169 \pm 6$ & $-11 \pm 2$ \\
\hline 33 & 2011-Feb-16 15:26 & $\mathrm{C} 7.7$ & $218 \pm 34$ & $5 \pm 34$ & $-212 \pm 48$ & $180 \pm 6$ & $163 \pm 6$ & $-16 \pm 5$ \\
\hline 34 & 2011-Feb-16 19:29 & $\mathrm{C} 1.3$ & $177 \pm 34$ & $135 \pm 34$ & $-41 \pm 48$ & $176 \pm 6$ & $170 \pm 6$ & $-6 \pm 3$ \\
\hline 35 & 2011-Feb-16 20:11 & C1.1 & $179 \pm 34$ & $89 \pm 34$ & $-89 \pm 48$ & $180 \pm 6$ & $180 \pm 6$ & $0 \pm 3$ \\
\hline 36 & 2011-Feb-16 21: 6 & $\mathrm{C} 4.2$ & $248 \pm 34$ & $107 \pm 34$ & $-141 \pm 48$ & $159 \pm 6$ & $158 \pm 6$ & $0 \pm 3$ \\
\hline
\end{tabular}


Table 3. Energy budget for the X2.2 flare on 2011-Feb-15. The magnetic energies labeld with the symbol *) refers to HMI data that were subjected to pre-processing (Sun et al. 2012a; Jiang and Feng 2013).

\begin{tabular}{|c|c|c|}
\hline $\begin{array}{l}\text { Energy } \\
\text { type }\end{array}$ & $\begin{array}{c}\text { Energy } \\
E\left(10^{30} \text { erg }\right)\end{array}$ & Reference \\
\hline \multicolumn{3}{|l|}{ Energy Input: } \\
\hline \multirow[t]{4}{*}{$\overline{\text { Total nonpotential energy } E_{N}}$} & $1210^{*}$ & Sun et al. $(2012 a)$ \\
\hline & $983-1090^{*}$ & Jiang and Feng (2013) \\
\hline & $492 \pm 158$ & Vemareddy et al. (2012b) \\
\hline & 857 & (This study) \\
\hline \multirow[t]{4}{*}{ Total potential energy $E_{P}$} & $970^{*}$ & Sun et al. (2012a) \\
\hline & $818-897^{*}$ & Jiang and Feng (2013) \\
\hline & $243 \pm 78$ & Vemareddy et al. (2012b) \\
\hline & 760 & (This study) \\
\hline \multirow[t]{3}{*}{ Free magnetic energy $E_{\text {free }}=E_{N}-E_{P}$} & 240 & Sun et al. 92012a) \\
\hline & $165-193$ & Jiang and Feng (2013) \\
\hline & $98 \pm 20$ & (This study) \\
\hline \multirow{2}{*}{ Decrease of free energy $E_{\text {post }}-E_{\text {pre }}$} & $37 \pm 4$ & Sun et al. (2012a) \\
\hline & $62 \pm 28$ & (This study) \\
\hline Minimum Current Corona (MCC) energy & 168 & Tarr et al.(2013) \\
\hline Energy drop from virial theorem & $105 \pm 104$ & Vemareddy et al. (2012b) \\
\hline Energy drop from virial theorem & 84 & Tziotziou et al. ( 2013) \\
\hline \multicolumn{3}{|l|}{ Energy output: } \\
\hline Nonthermal energy RHESSI (25-50 keV) & 54 & Sun et al. (2012a) \\
\hline Thermal energy AIA/SDO & 20 & Aschwanden et al. (2013a) \\
\hline Radiated energy in Ly alpha & 1.0 & Milligan et al. (2012) \\
\hline Radiatied in free-free continuum & 0.8 & Milligan et al. (2012) \\
\hline Radiated in GOES X-rays 1-8 A & 0.5 & Milligan et al. (2012) \\
\hline Radiated in Lyman continuum & 0.4 & Milligan et al. (2012) \\
\hline Radiated in He I 304 A & 0.3 & Milligan et al. (2012) \\
\hline Radiated in He I cont & 0.04 & Milligan et al. (2012) \\
\hline Radiated in He II cont & 0.01 & Milligan et al. (2012) \\
\hline Helioseismic energy in $3-4 \mathrm{mHz}$ band & 0.0018 & Alvarado-Gomez et al. (2012) \\
\hline
\end{tabular}


Table 4. Energy budget of X,M-class flares during 2011 Feb 12-16: $\Delta E_{\text {free }}^{a}=$ change of free energy calculated with COR-NLFFF code in this study; $\Delta E_{\text {free }}^{b}=$ change of free energy calculated with the photospheric (Wiegelmann) NLFFF code (Sun et al. 2012a); $\Delta E_{M C C}=$ energy change from Minimum Current Corona model (Tarr et al. 2013); $\Delta E_{V T}^{c}=$ energy change from virial theorem (Vemareddy et al. 2012b); $\Delta E_{V T}^{d}=$ energy change from virial theorem (Tziotziou et al. 2013); $E_{t h}=$ thermal energy from AIA/SDO DEM analysis (Aschwanden et al. 2013a).

\begin{tabular}{|c|c|c|c|c|c|c|c|c|}
\hline $\begin{array}{c}\text { Flare } \\
\#\end{array}$ & $\begin{array}{c}\text { Observation } \\
\text { Date }\end{array}$ & $\begin{array}{c}\text { GOES } \\
\text { class }\end{array}$ & $\begin{array}{c}\Delta E_{\text {free }}^{a} \\
\left(10^{32} \text { erg }\right)\end{array}$ & $\begin{array}{c}\Delta E_{\text {free }}^{b} \\
\left(10^{32} \text { erg }\right)\end{array}$ & $\begin{array}{c}\Delta E_{M C C} \\
\left(10^{32} \mathrm{erg}\right)\end{array}$ & $\begin{array}{c}\Delta E_{V T}^{c} \\
\left(10^{32} \mathrm{erg}\right)\end{array}$ & $\begin{array}{c}\Delta E_{V T}^{d} \\
\left(10^{32} \text { erg }\right)\end{array}$ & $\begin{array}{c}\Delta E_{t h} \\
\left(10^{32} \mathrm{erg}\right)\end{array}$ \\
\hline 2 & 2011-Feb-13 17:27 & M6.6 & $-44 \pm 9$ & $-21 \pm 2$ & -4 & $-43 \pm 17$ & -10 & 25 \\
\hline 11 & 2011-Feb-14 17:20 & $\mathrm{M} 2.2$ & $-62 \pm 28$ & $-16 \pm 3$ & -3 & & -49 & \\
\hline 16 & 2011-Feb-15 1:43 & $\mathrm{X} 2.2$ & $-68 \pm 20$ & $-37 \pm 4$ & -168 & $-105 \pm 104$ & -84 & 20 \\
\hline 24 & 2011-Feb-16 1:31 & M1.0 & $-126 \pm 48$ & $0 \pm 2$ & & & & 3 \\
\hline 28 & 2011-Feb-16 7:34 & M1.1 & $-123 \pm 48$ & $0 \pm 4$ & & & & 2 \\
\hline 32 & 2011-Feb-16 14:18 & M1.6 & $-157 \pm 48$ & $-11 \pm 2$ & & & -65 & 5 \\
\hline
\end{tabular}




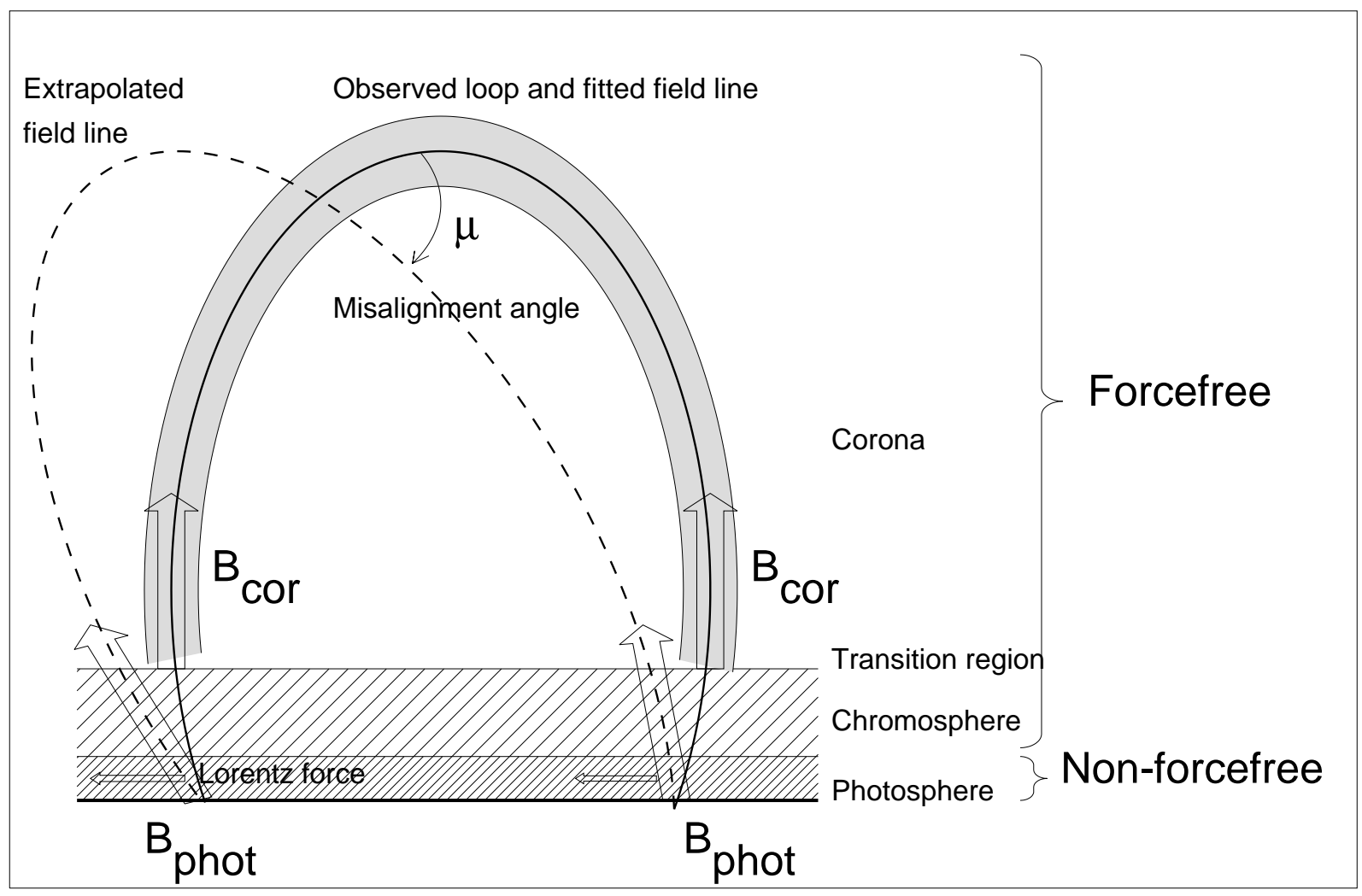

Fig. 1. - The diagram shows the misalignment between a magnetic field line (dashed curve) that is extrapolated from the magnetic field vectors $\mathbf{B}_{\text {phot }}$ from the non-forcefree photosphere, and a magnetic field line (solid curve) that is obtained from forward-fitting of a nonlinear forcefree field model $\mathbf{B}_{\text {cor }}$ to an observed loop geometry (grey color), quantified by a misalignment angle $\mu$. 


\section{Coronal Non-Linear Force-Free Field Forward-Fitting Code (COR-NLFFF)}

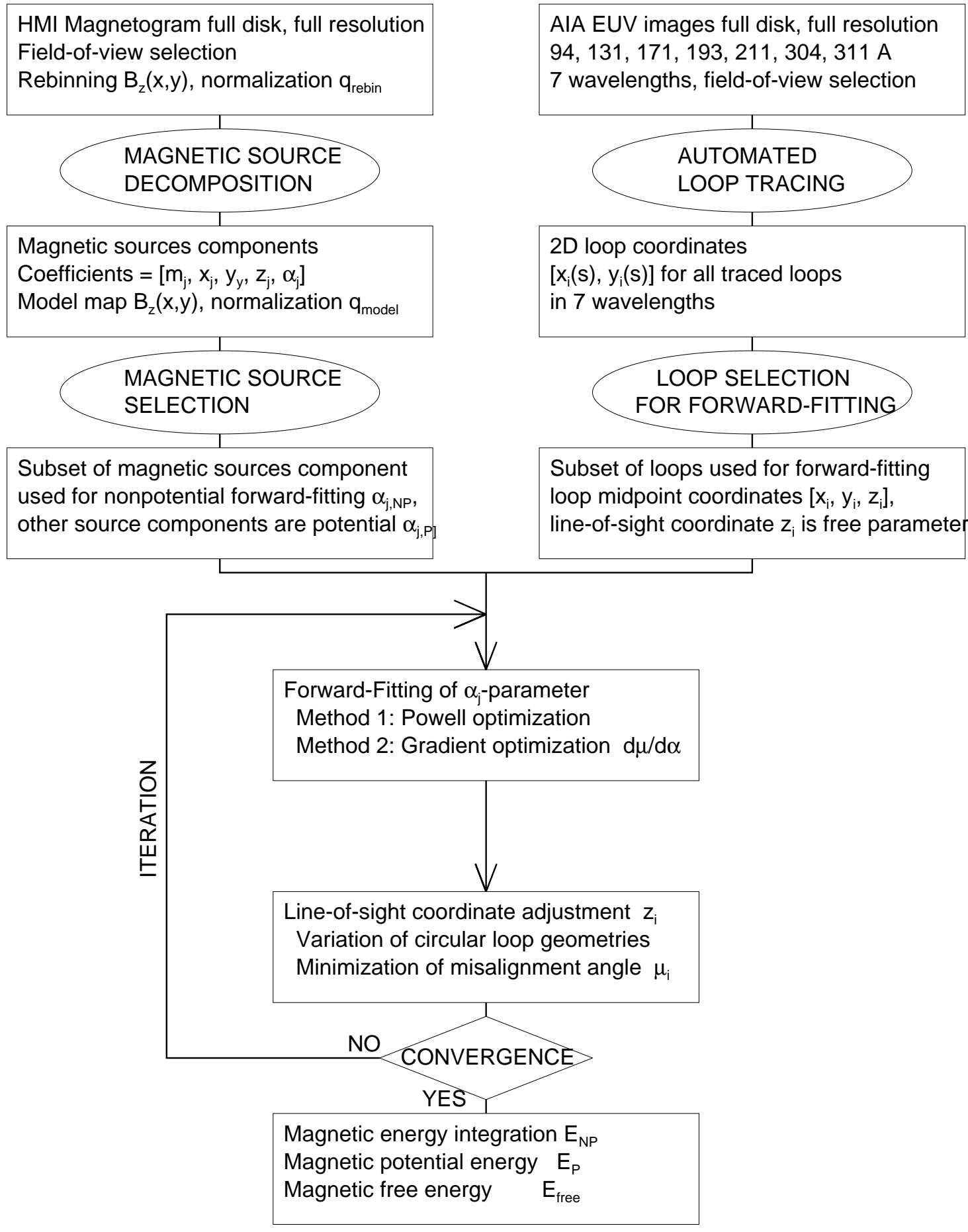

Fig. 2.- Flow chart of the COR-NLFFF code, which includes processing of the magnetic data (top left), of the EUV image data (top right), and forward-fitting using both data sets (bottom half). See Section 2 for a theoretical description, and Section 3 and Appendix A for parametric tests. 

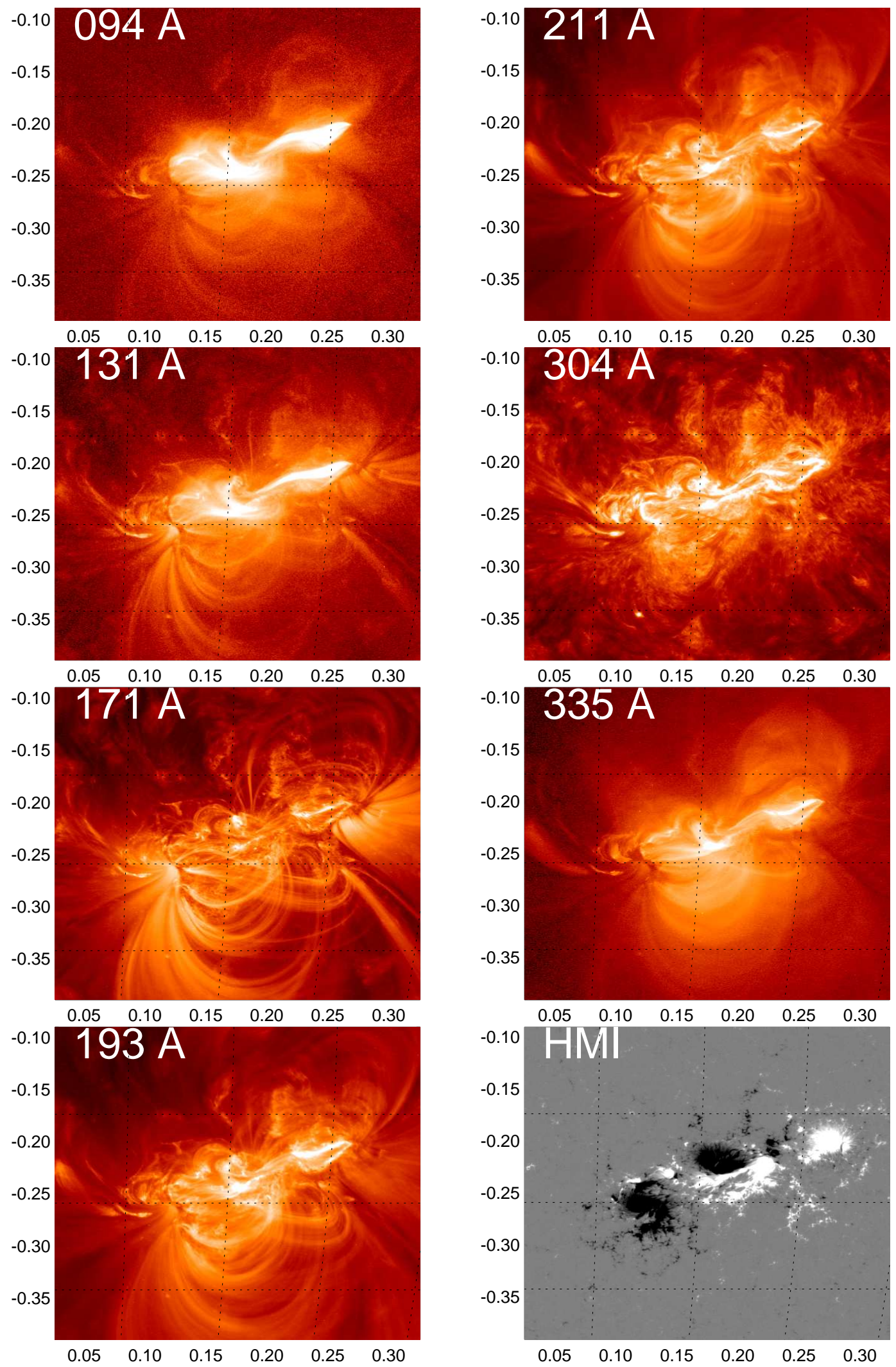

Fig. 3.- A set of 7 EUV images observed with AIA/SDO between 2011-02-15 00:00:00 UT and 00:00:12 UT, in the wavelengths of $94,131,171,193,211,304,335 \AA$, rendered on a logarithmic color scale. The co-spatial and cotemporanous HMI/SDO magnetogram (bottom right) was observed on 2011-02-14 23:58:57 $\mathrm{UT}$, rendered in greyscale. The field-of-view of all images is $x=[0.03-0.33]$ solar radii in EW direction and $y=[-0.39,-0.09]$ solar radii in NS direction, corresponding to a range of $210 \mathrm{Mm}$. 

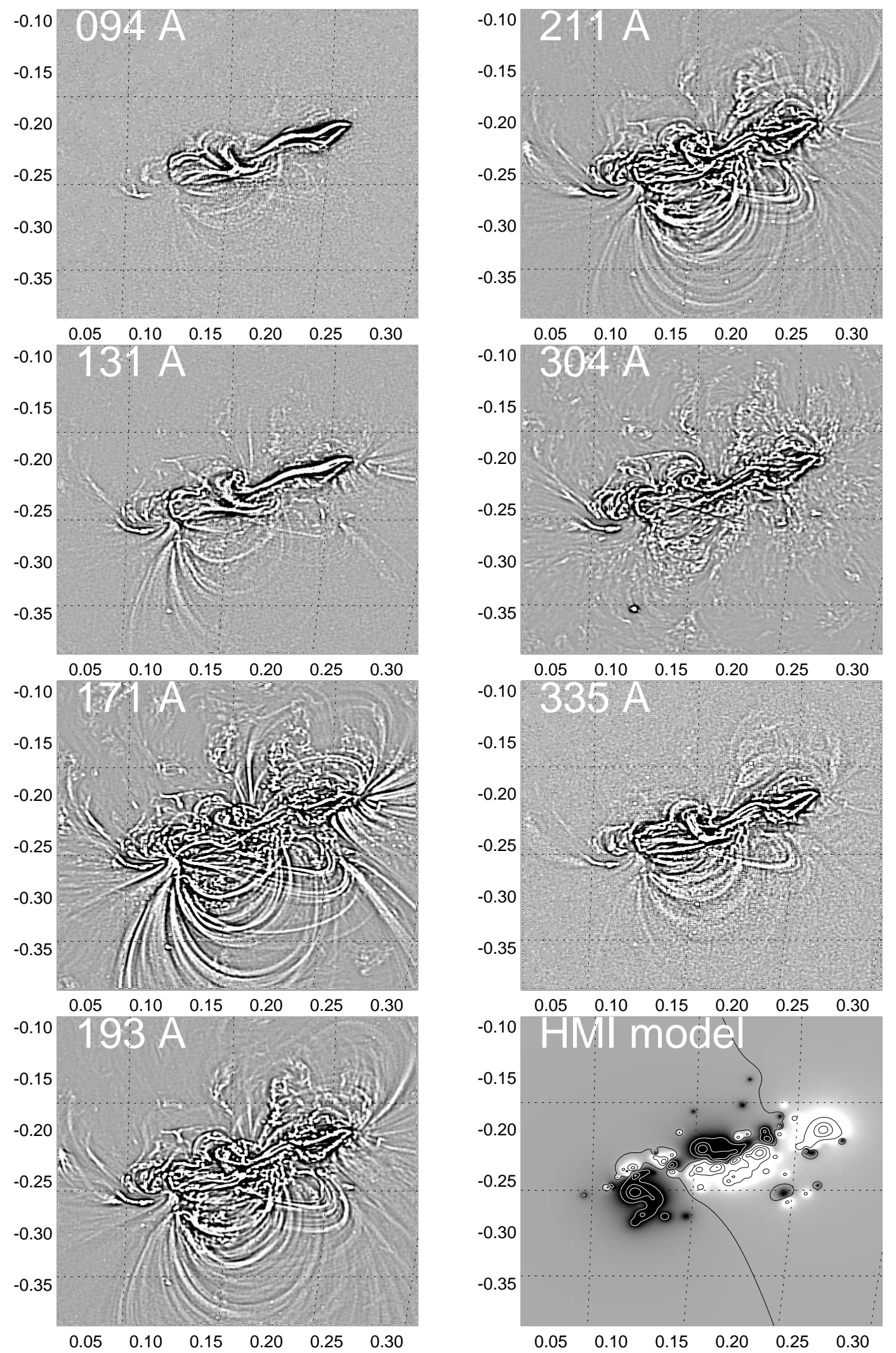

Fig. 4.- The same dataset of AIA images shown in Fig. 3 is bandpass-filtered with a lowpass filter boxcar of $n s m_{1}=5$ pixels and a highpass filter boxcar of $n s m_{2}=7$ pixels. The HMI magnetogram (bottom right) is decomposed into 100 Gaussian-like magnetic source components and superimposed to a LOS model map that is parameterized with $4 \times 100$ parameters. 

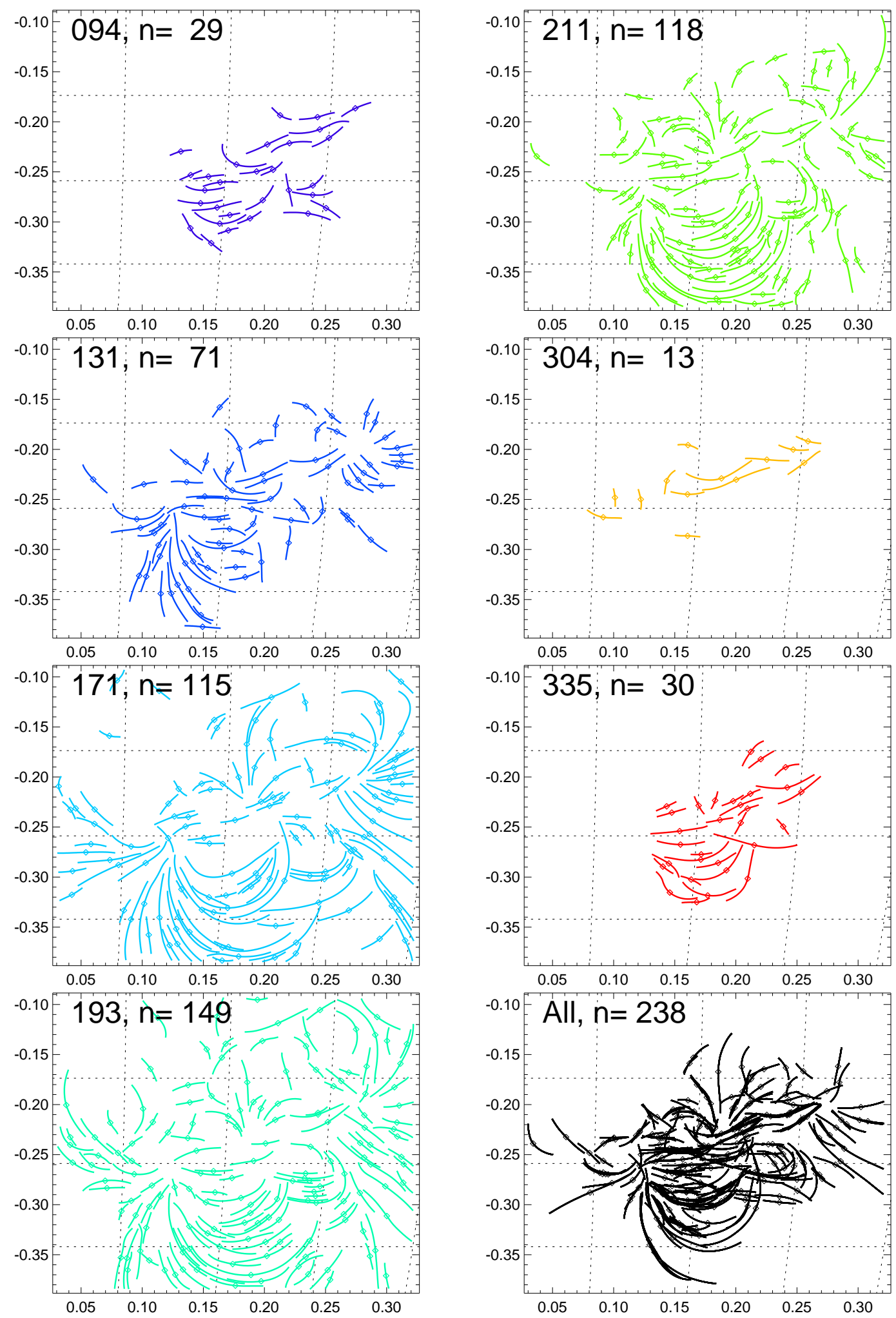

Fig. 5.- Automated loop tracing of the 7 bandpass-filtered AIA images (shown in Fig. 4) with the code OCCULT-2. Each wavelength is represented with a different color, and a composite of all wavelengths (containing a limit of 50 loop structures per wavelength) is shown in the bottom right panel. The number $n_{\text {loop }}$ of loop segments detected above a noise threshold and with a minimum length of 30 pixels is indicated. 

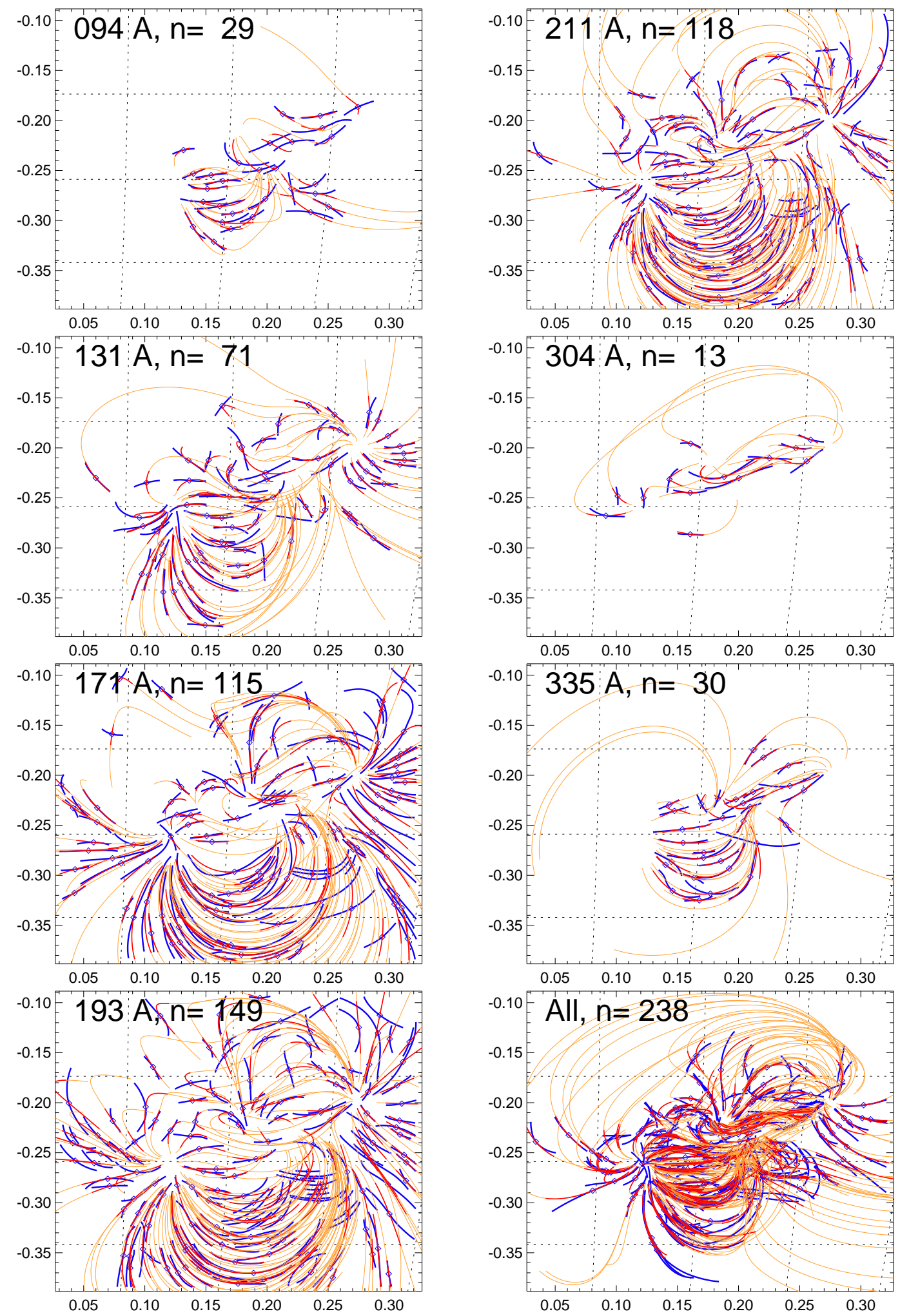

Fig. 6.- Forward-fitting of NLFFF magnetic field approximation (orange curves) to the automatically traced loops (blue curves) in each wavelength filter. The locations of the theoretical field lines where chosen at the intersection of the midpoints (diamonds) of traced loops, and a field line segment of equal length as the traced loop segment is shown with a red curve. The bottom right panel shows a simultaneous fit to a subset of $n=238$ loops synthesized from the 7 AIA filters. 

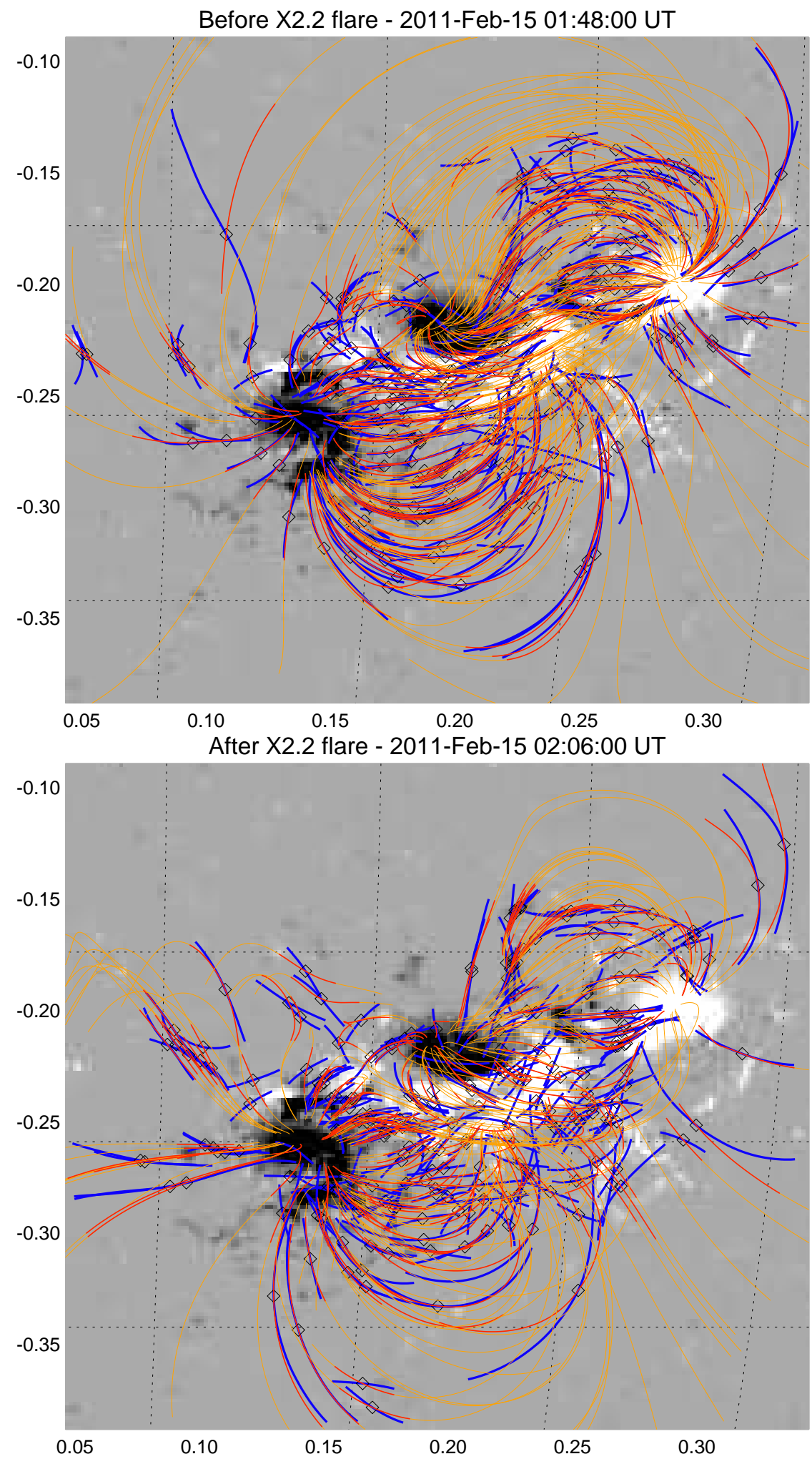

Fig. 7.- Best-fit solution of nonlinear force-free field (orange curves) overlaid on the automatically traced coronal loops (blue curves), tailored to the same loop length segments (red curves), before the X2.2 flare on 2011-Feb-15, 01:48 UT (top panel), and after the flare peak on 2011-Feb-15, 02:06 UT (bottom panel), overlaid on the line-of-sight HMI magnetogram. The time range covers the interval of the largest energy decrease of free magnetic energy during the flare. 


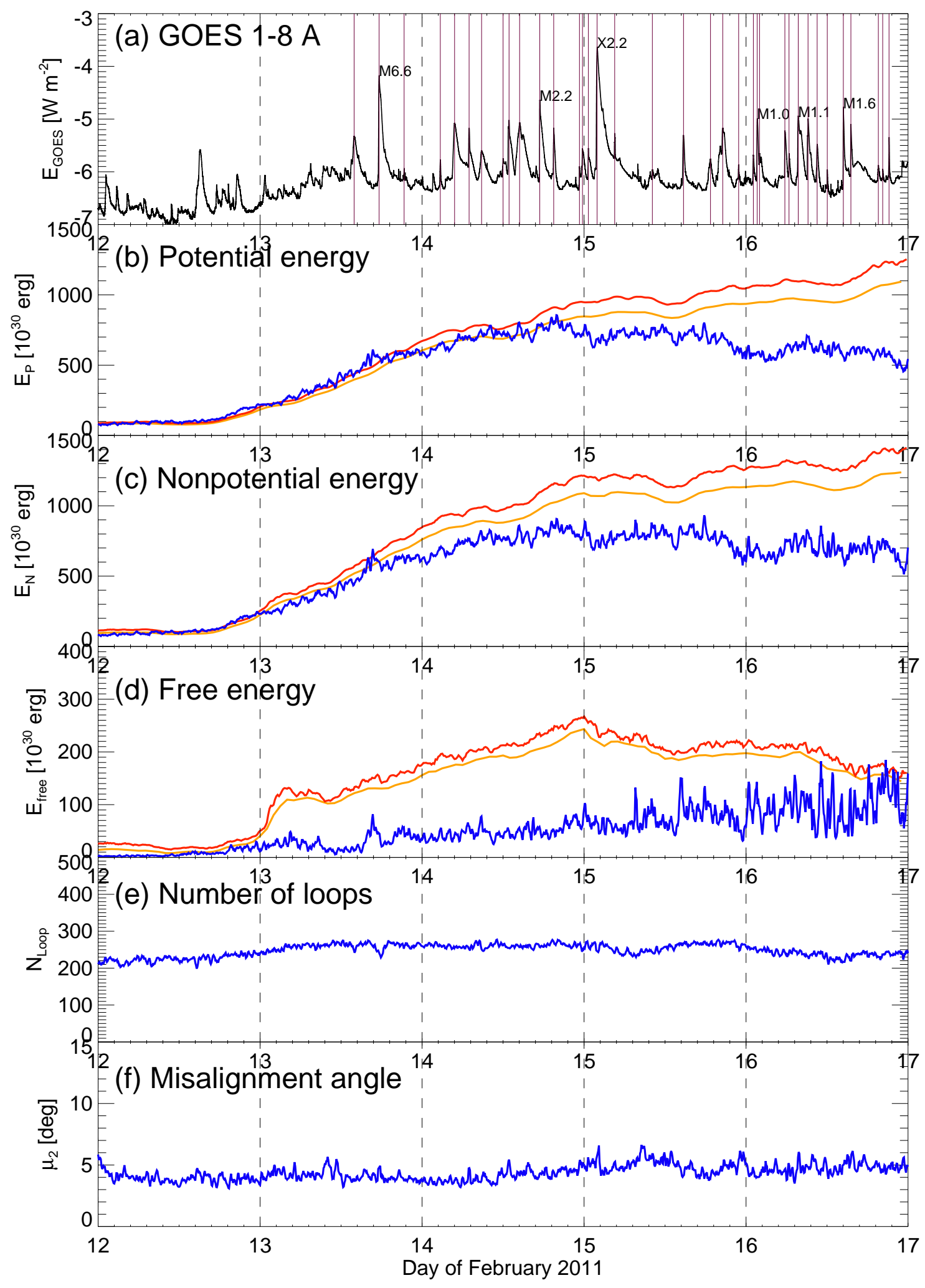

Fig. 8. - Time evolution of magnetic energies of AR 11158 during 2011 Feb 12 to 17: (a) GOES 1-8 $\AA$ flux, with GOES C-, M-, and X-class flares indicated with purple vertical lines; (b) Potential field energy $E_{P}$; (c) Nonpotential energy $E_{N}$; (d) Free energy $E_{\text {free }}=E_{N}-E_{P}$; (e) The number of fitted loops $N_{\text {loop }}$; (f): the 2D misalignment angle $\mu_{2}$ of the best fit. The color code indicates forward-fitting of traced loops with the COR-NLFFF code in 6-min time intervals (blue), the Wiegelmann NLFFF code in 12-min intervals (red), 

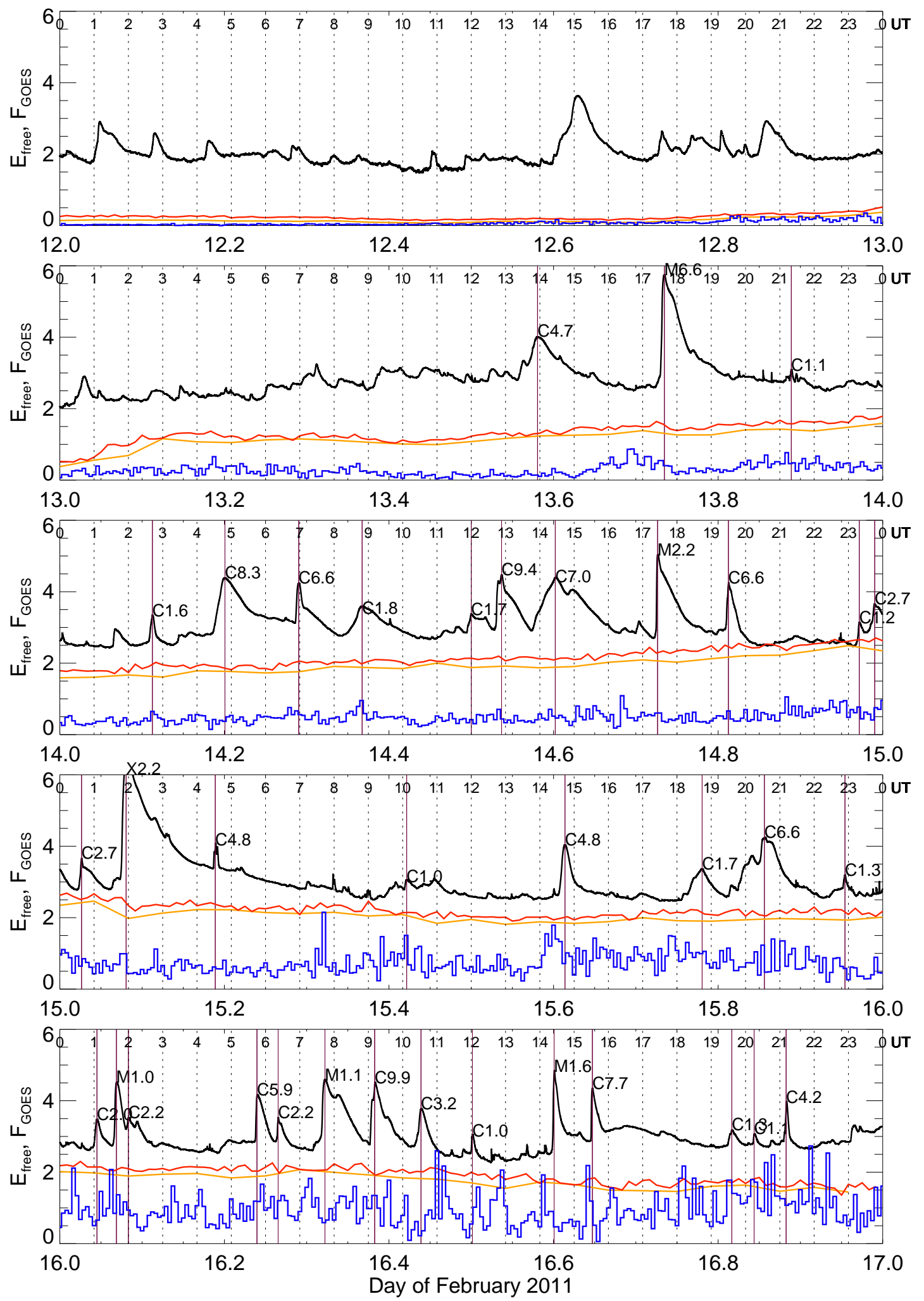

Fig. 9.- Expanded time profiles of the GOES 1-8 A flux (black, arbitrary units), the free energies computed with the Wiegelmann code in 12-min intervals (red) and 1-hr intervals (orange; Sun et al. 2012a), with forward-fitting of automatically traced loops in 6-min intervals (blue). The times of 36 GOES C-,M-, and $\mathrm{X}$-class flares occurring in AR 11158 are indicated with vertical purple lines and labeled with the GOES class. Each panel represents a consecutive day from 2011 Feb 12 to 17 . 
Flare \# 2

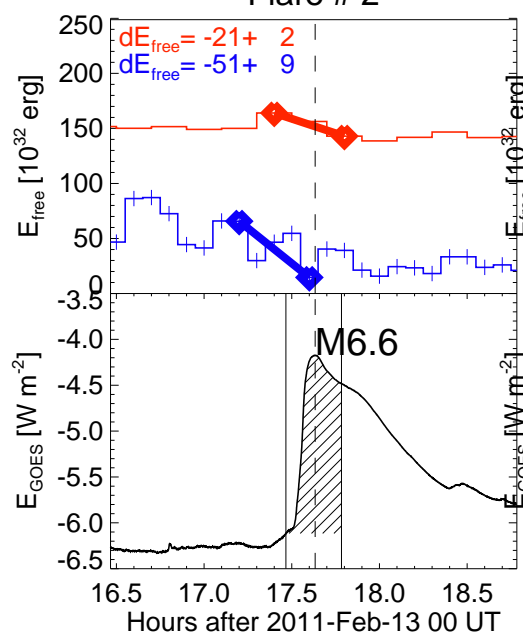

Flare \#19

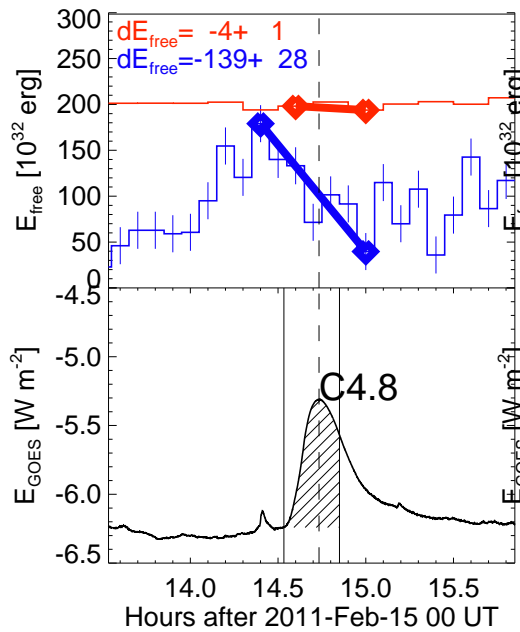

Flare \#29

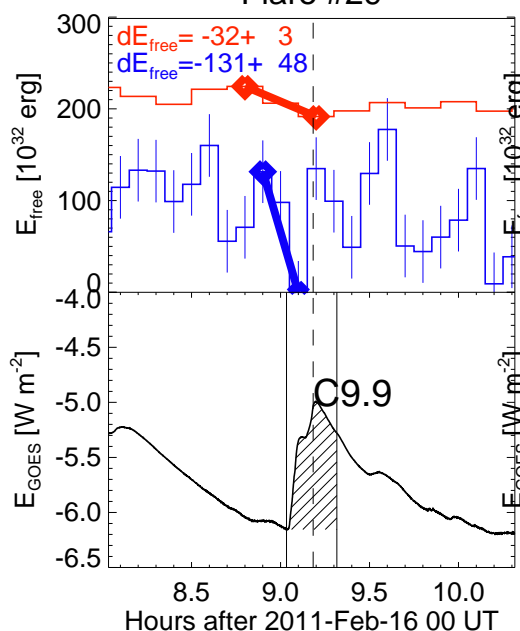

Flare \#11

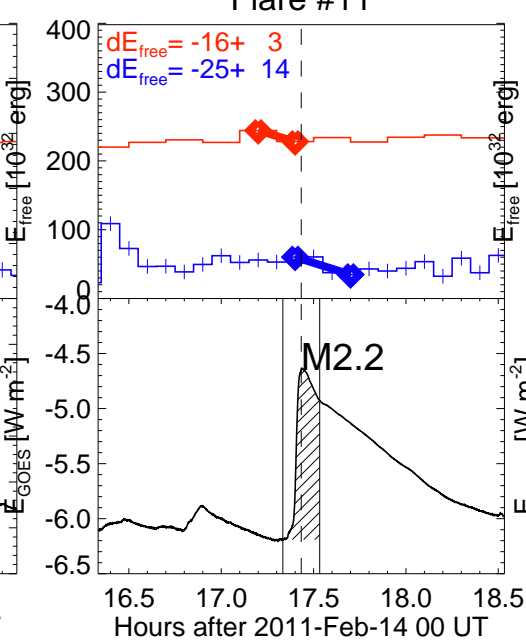

Flare \#24

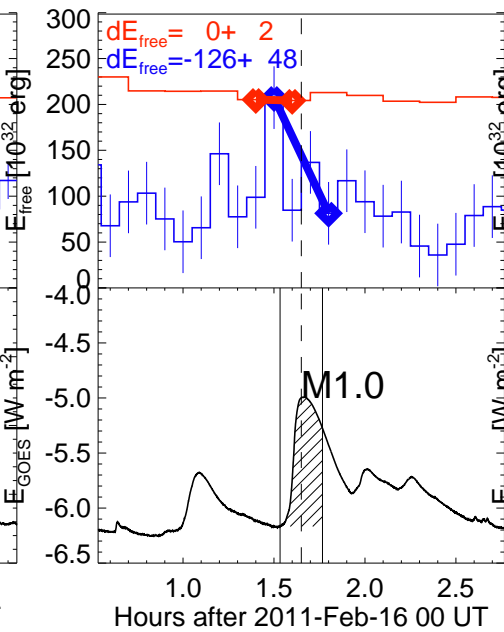

Flare \#32

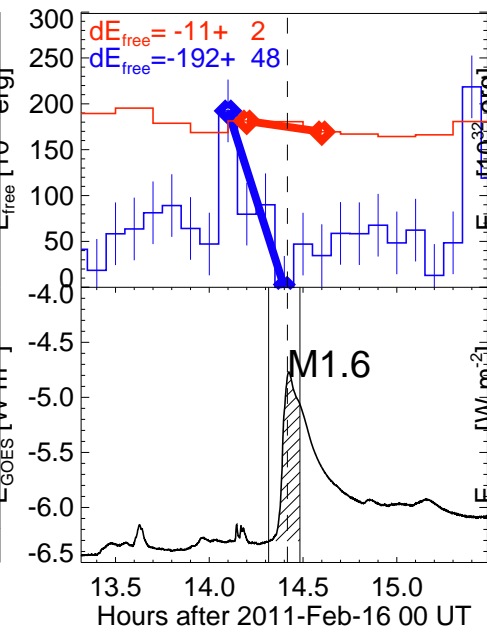

Flare \#16

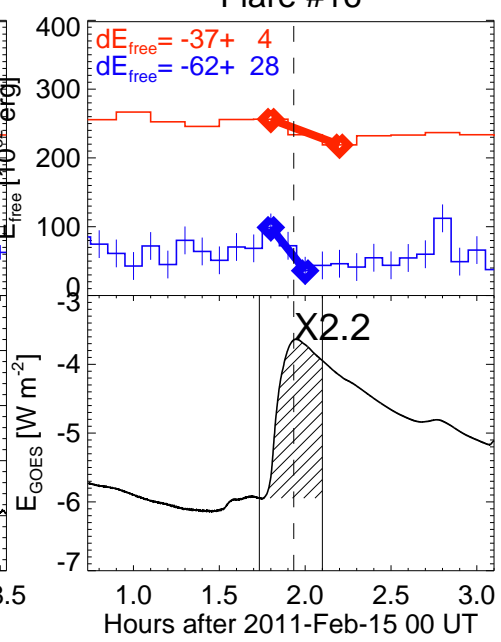

Flare \#28

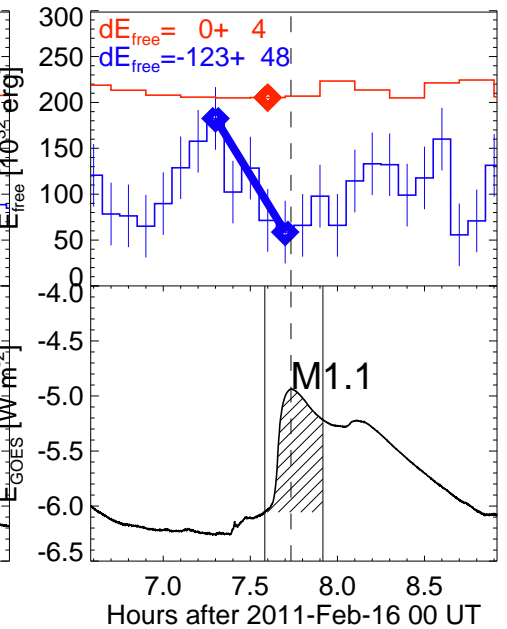

Flare \#33

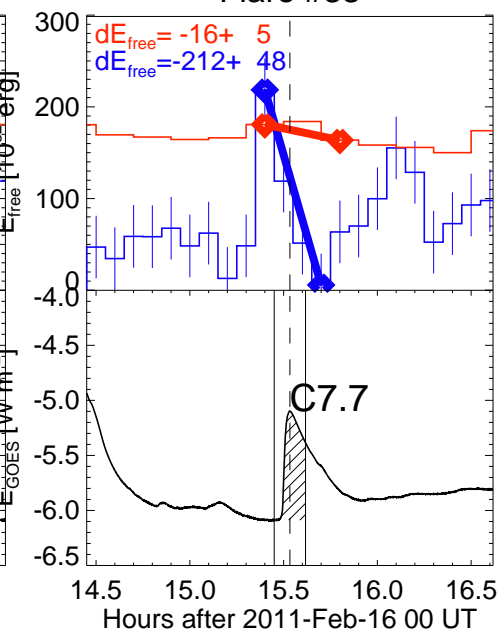

Fig. 10.- The change in free magnetic energy is shown during 9 flares (shown in 9 different panels), including the GOES 1-8 A flux (black), bracketed between start $\left(t_{\text {start }}\right)$ and end time $\left(t_{\text {end }}\right)$ (hatched between vertical lines), and peaking at $t_{\text {peak }}$ (dashed vertical line), the free energy $E_{\text {free }}$ computed with forward-fitting to coronal loops (blue histrograms), and with the Wiegelmann NLFFF code (red histrograms). The decrease of the free energy is measured between the maximum free energy in the preflare interval $\left[t_{\text {start }}-0.3 \mathrm{hr}, t_{\text {neak }}\right]$ 


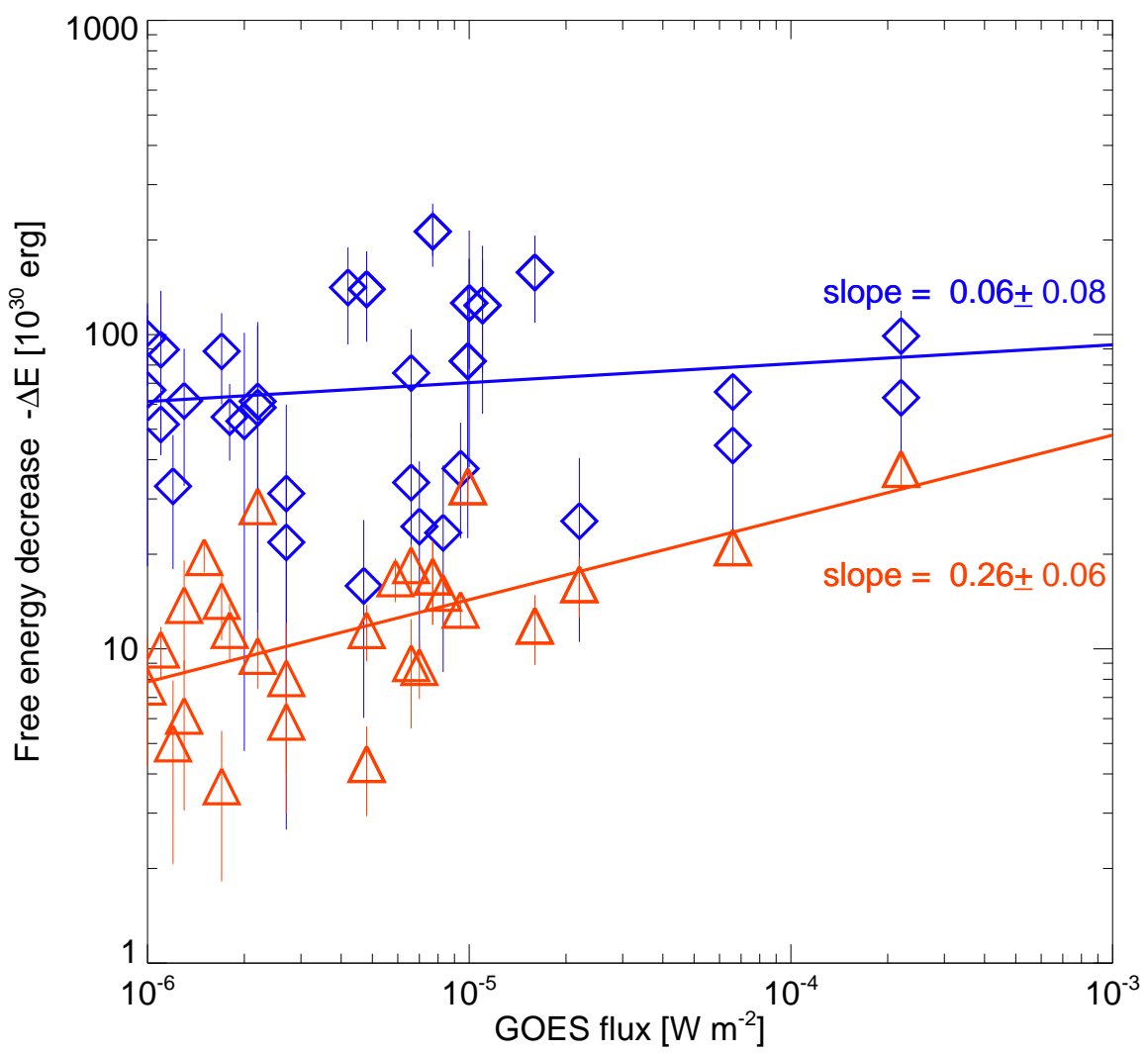

Fig. 11. - Scatterplot of the decrease of free magnetic energy $\left(-\Delta E_{\text {free }}\right)$ with the GOES flux $\left[\mathrm{W} \mathrm{m} \mathrm{m}^{-2}\right]$ for all analyzed flare events with significant decreases. The energy drops calculated with the Wiegelmann NLFFF code are indicated with red triangles, and those with the COR-NLFFF code with blue diamonds. Linear regression fits are indicated. Note the order of magnitude difference in energy decreases between the two codes. 

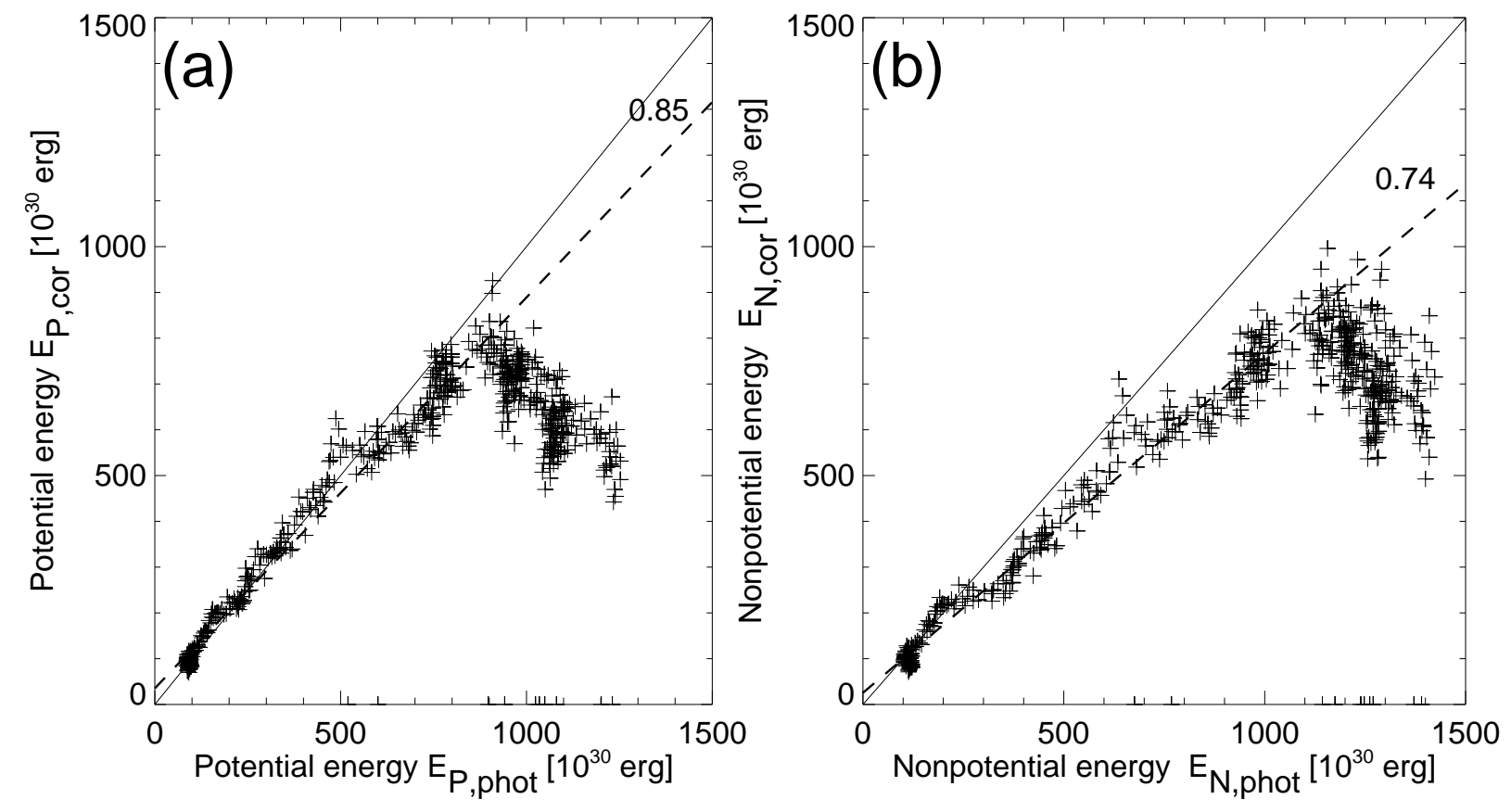

Fig. 12.- Correlation of the potential energy $E_{P}$ (left panel) and the free energy $E_{\text {free }}=E_{N}-E_{P}$ (right panel) for the two codes, i.e., the Wiegelmann NLFFF code (y-axis) and the COR-NLFFF code using forward-fitting to coronal loops (x-axis). 


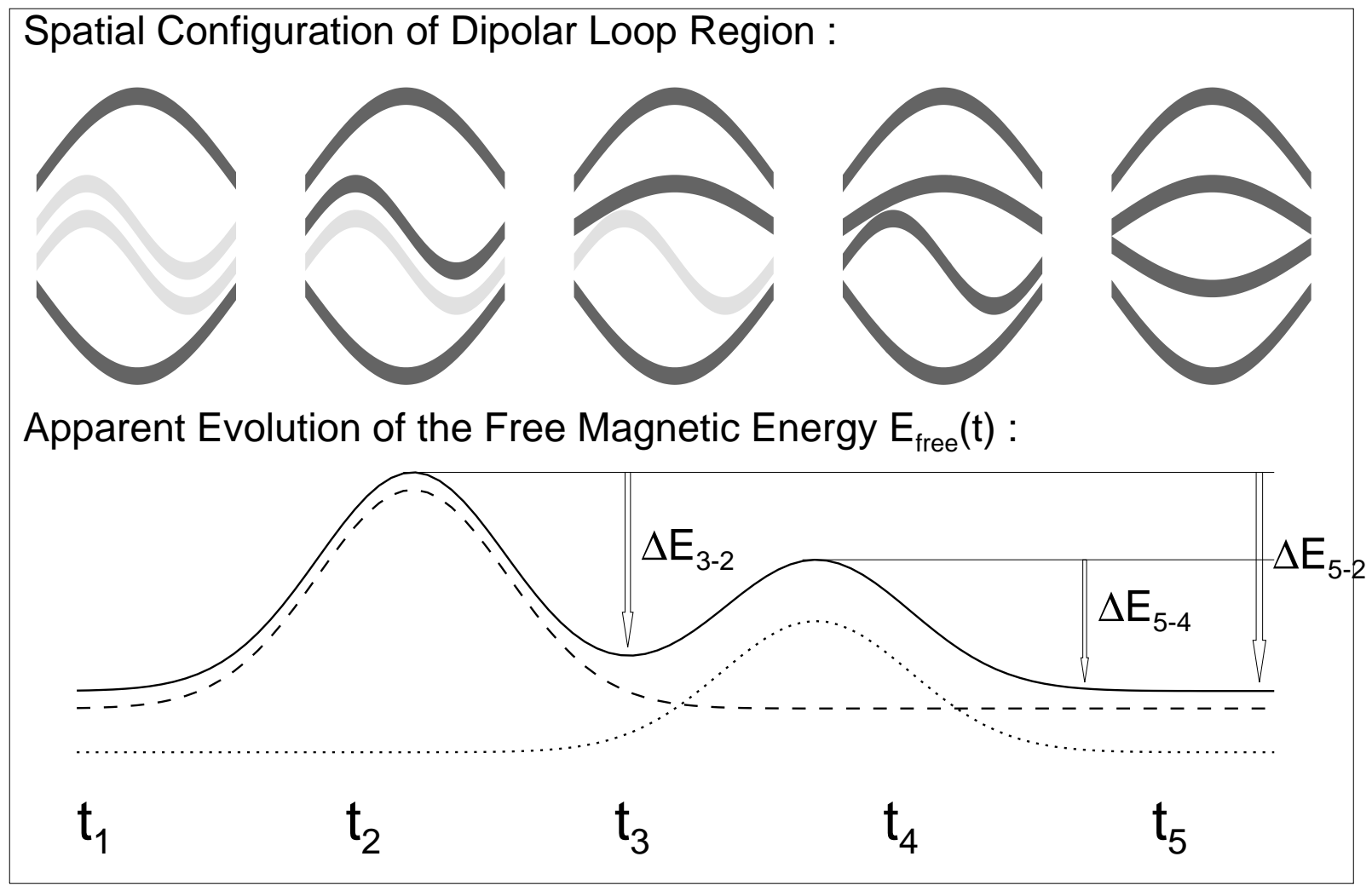

Fig. 13. - Schematic diagram of the spatial loop configuration (top panel) and evolution of free magnetic energy $E_{\text {free }}(t)$ during a flare. Mostly potential loops are visible at the beginning of a flare $\left(t_{1}\right)$, while a first sigmoid is illuminated at $t_{2}$, which relaxes to a potential loop at atim $t_{3}$. A second sigmoid is illuminated at time $t_{4}$, which relaxes to a potential loop at time $t_{5}$. The total energy difference before and after the flare, $\Delta E_{5-2}$, is a lower limit to the sum of all sequential energy releases $\Delta E_{3-2}$ and $\Delta E_{5-4}$, and thus underestimates the total dissipated magnetic energy. 

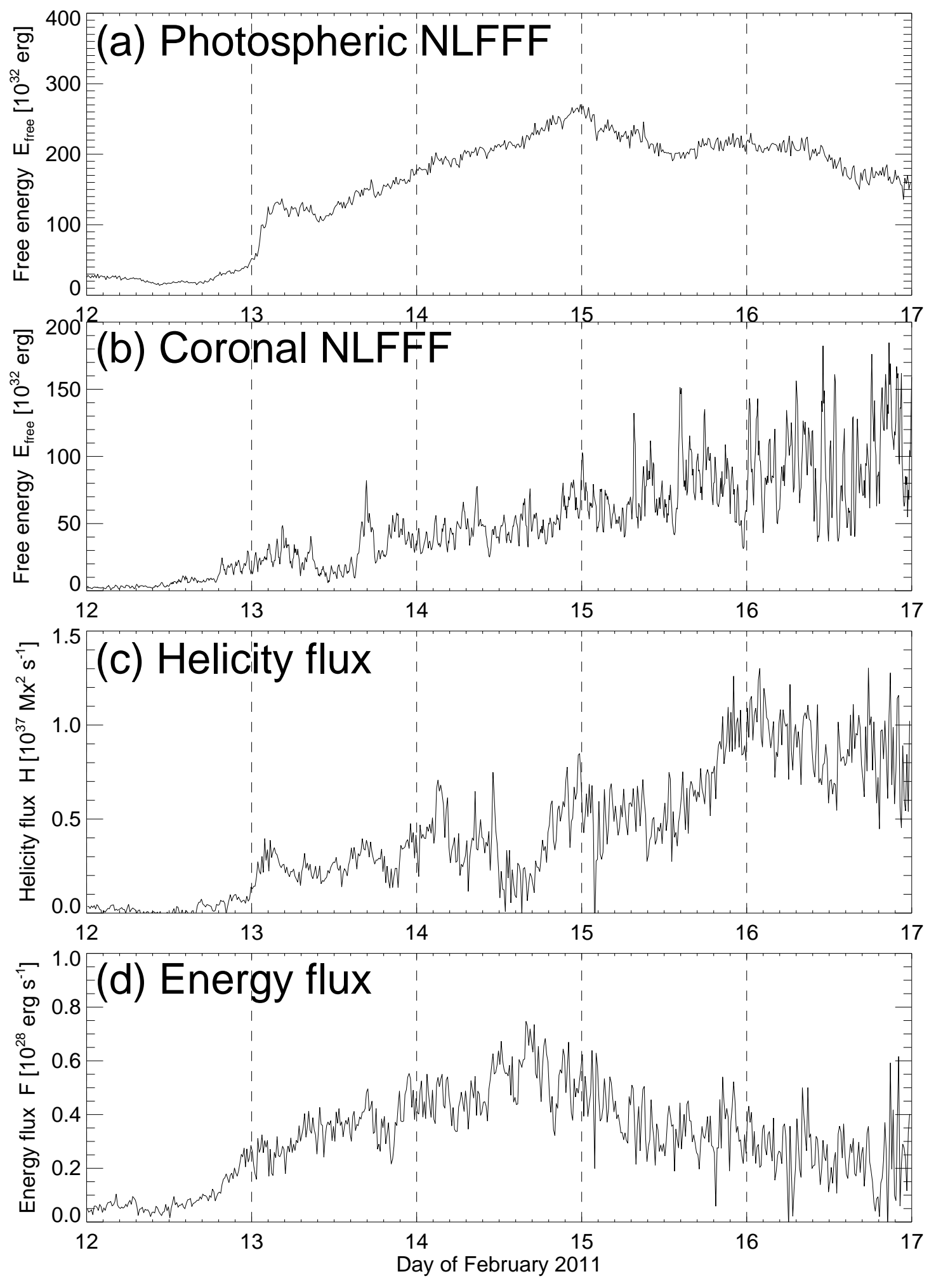

Fig. 14.- Comparison of the free energy calculated with the photospheric NLFFF code (a), with the coronal NLFFF code (b), the helicity flux (c), and the energy flux (d). Note that the photospheric NLFFF code produces very little variability, while the coronal NLFFF code has a much higher degree of variability. 

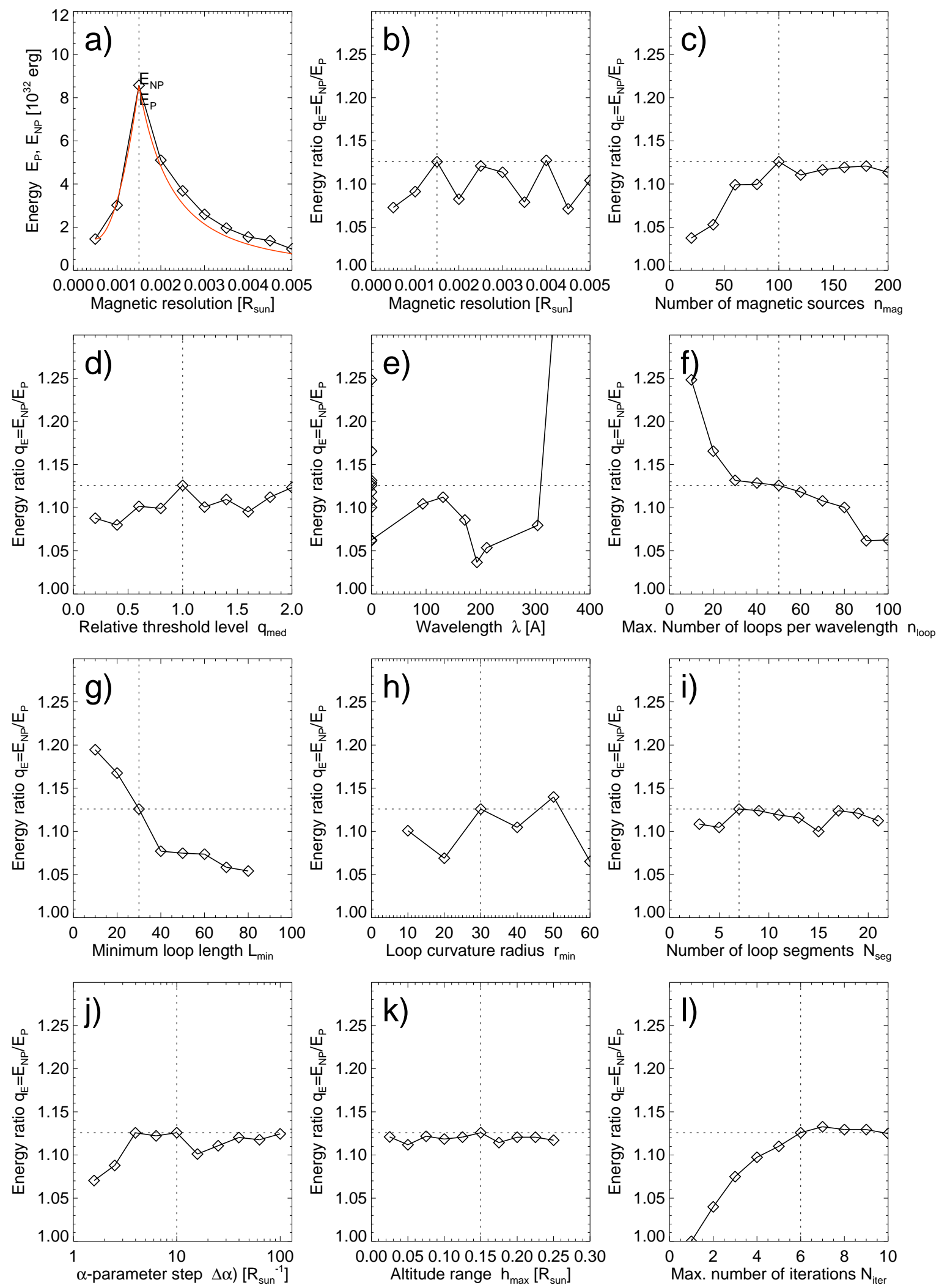

Fig. 15.- (a) The nonpotential energy $E_{N}$ and potential energy $E_{P}$ as a function of the magnetic resolution $\Delta x_{m a g}$, along with a theoretical model (red curves) explained in Section 3.1. The panels (b) through (l) contain 11 parametric studies of the energy ratio $q_{E}=E_{N} / E_{P}$ (diamonds) as a function of 11 control parameters varied over some range. The vertical dotted lines indicate the chosen default values, and the horizontal dotted lines indicate the energy ratio at the default value. See full description in Appendix A. 

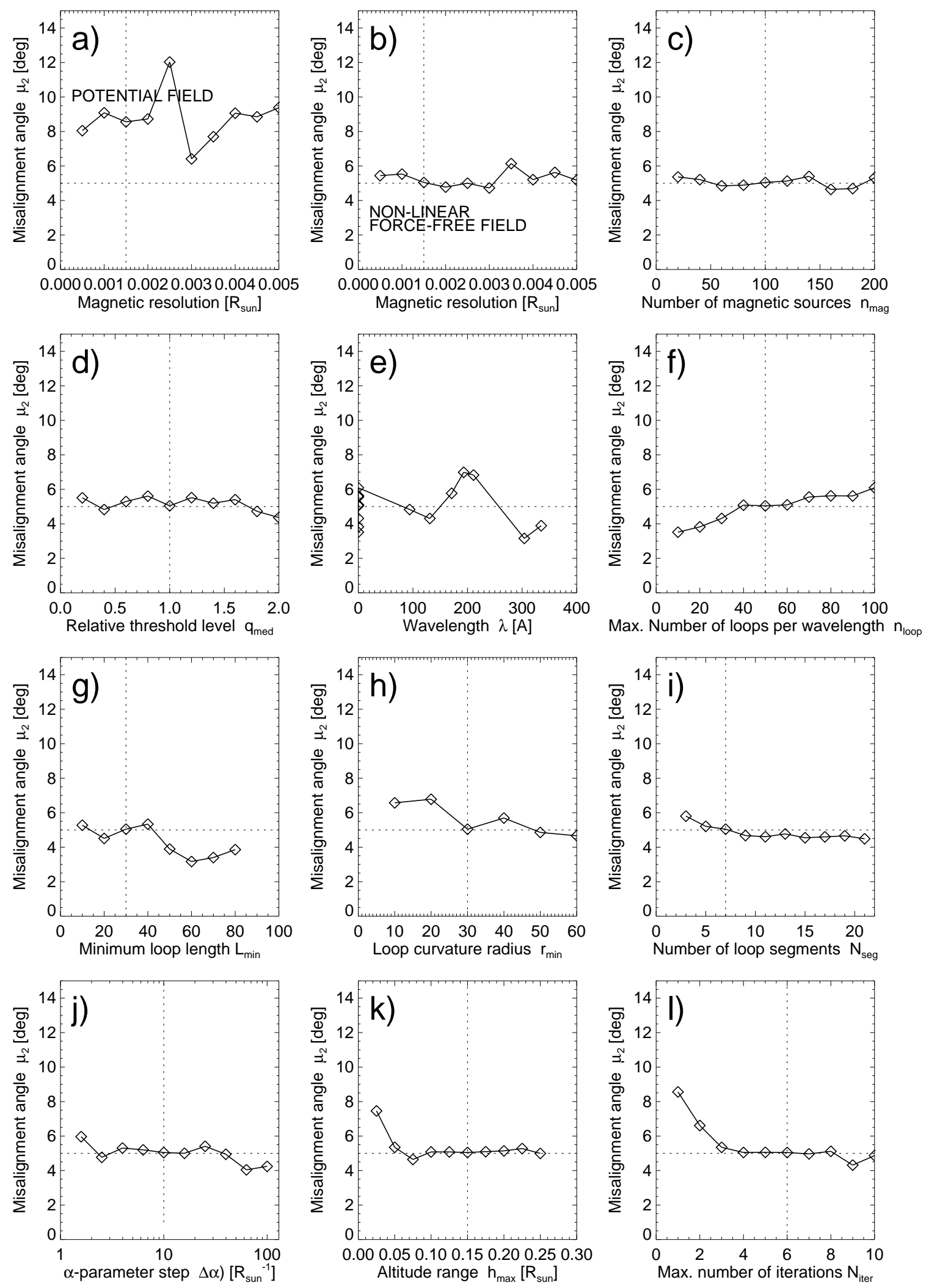

Fig. 16.- (a) Misalignment angle $\mu_{2}$ for the potential field as a function of the magnetic resolution from 1 to 10 HMI pixels. The panels (b) through (l) contain 11 parametric studies of the misalignment angle $\mu_{2}$ (diamonds) as a function of 11 control parameters varied over the same range as in Fig. 11. The vertical dotted lines indicate the chosen default values, and the horizontal dotted lines indicate the misalignment angle at the default value. 

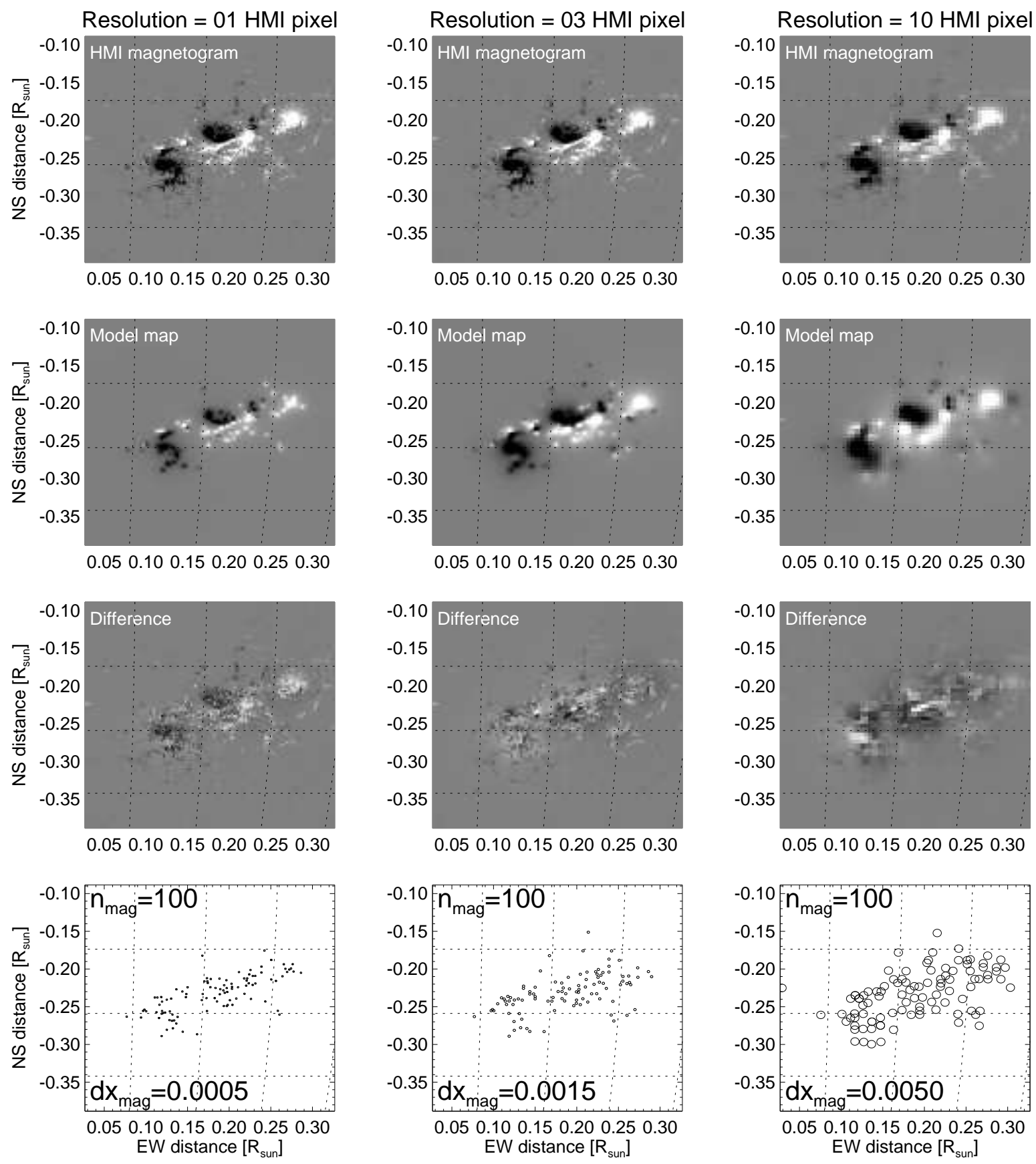

Fig. 17.- Original HMI magnetogram $B_{z}(x, y)$ with full resolution (top left), rebinned with 3 HMI pixels (top middle), and rebinned with $10 \mathrm{HMI}$ pixels (top right). The magnetograms are decomposed with the three different resolutions into 100 magnetic sources that form the model map (second row). The difference between the observed magnetograms and the model maps are shown in the third row with identical greyscale. The location and radii are shown in the bottom row. 